\title{
STK4 deficiency underlies impaired interferon signaling and T cell immunity
}

Andrea Guennoun

Sidra Medicine

Salim Bougarn

Sidra Medicine

Taushif Khan

Sidra Medicine

Rafah Mackeh

Sidra Medicine

Mahbuba Rahman

Sidra Medicine

Fatima Al Ali

Sidra Medicine

Manar Ata

Sidra Medicine

Waleed Aamer

Sidra Medicine

Debra Prosser

Sidra Medicine

Tanwir Habib

Sidra Medicine

Evonne Chin-Smith

Sidra Medicine

Khawla Al-Darwish

Sidra Medicine

Qian Zhang

The Rockefeller University

\section{Alya Al-Shakaki}

Weill Cornell Medicine-Qatar

\section{Amal Robay}

Weill Cornell Medicine-Qatar

Ronald Crystal

Weill Cornell Medicine

Khalid Fakhro 
Sidra Medicine

Amal Al-Naimi

Sidra Medicine

Eman Al Maslamani

Sidra Medicine

Amjad Tuffaha

Sidra Medicine

Ibrahim Janahi

Sidra Medicine

Mohammad Janahi

Sidra Medicine

Donald Love

Sidra Medicine

Mohammed Yousuf Karim

Sidra Medicine

Bernice Lo

Sidra Medicine

Amel Hassan

Sidra Medicine

Mehdi Adeli

Sidra Medicine

Nico Marr ( $\sim$ nmarr@sidra.org )

Sidra Medicine https://orcid.org/0000-0002-1927-7072

\section{Research Article}

Keywords: Human serine/threonine kinase 4 (STK4) deficiency, combined immunodeficiency, T cell lymphopenia, interferon, antibody repertoire, transcriptomics

Posted Date: March 22nd, 2021

DOl: https://doi.org/10.21203/rs.3.rs-328827/v1

License: (c) (1) This work is licensed under a Creative Commons Attribution 4.0 International License. Read Full License 
Andrea Guennoun ${ }^{\S,}$, Salim Bougarn ${ }^{\S,}$, Taushif Khan ${ }^{1}$, Rafah Mackeh ${ }^{1}$, Mahbuba Rahman, Fatima Al-Ali ${ }^{1}$, Manar Ata ${ }^{1}$, Waleed Aamer ${ }^{1}$, Debra Prosser, Tanwir Habib, Evonne ChinSmith ${ }^{1}$, Khawla Al-Darwish, Qian Zhang ${ }^{3}$, Alya Al-Shakaki ${ }^{4}$, Amal Robay $^{4}$, Ronald Crystal ${ }^{5}$, Khalid Fakhro ${ }^{1,4,6}$, Amal Al-Naimi ${ }^{7}$, Eman Al Maslamani ${ }^{7}$, Amjad Tuffaha, Ibrahim Janahi ${ }^{7}$, Mohammad Janahi ${ }^{7}$, Donald R. Love ${ }^{\psi, 2}$, Mohammed Yousuf Karim ${ }^{\psi 2}$, Bernice Lo ${ }^{\psi 1,6}$, Amel Hassan $^{\psi, 7}$, Mehdi Adeli ${ }^{\psi, 7}$, Nico Marr ${ }^{1,6}$ (ORCID: 0000-0002-1927-7072)

1 Research Branch, Sidra Medicine, Doha, Qatar

2 Department of Pathology, Sidra Medicine, Doha, Qatar

3 St. Giles Laboratory of Human Genetics of Infectious Diseases, Rockefeller Branch, The Rockefeller University, New York, NY, USA

4 Weill Cornell Medicine-Qatar, Doha, Qatar

5 Weill Cornell Medicine, New York, USA

6 College of Health and Life Sciences, Hamad bin Khalifa University, Doha, Qatar

7 Department of Pediatrics, Sidra Medicine, Doha, Qatar

$\S, \Psi$, equal contribution

Address correspondence to: Nico Marr, Sidra Medicine, Research Branch, Doha, Qatar, PO 
27 Purpose. Human serine/threonine kinase 4 (STK4) deficiency is a rare, autosomal recessive genetic disorder leading to combined immunodeficiency. The extent to which STK4 deficiency impairs immune signaling and host defenses is unclear. We assessed the functional consequences of a novel, homozygous nonsense STK4 mutation (NM_006282.2:c.871C>T, p.Arg291*) found in a pediatric patient by comparing the patient's innate and adaptive cellmediated and humoral immune responses with those of three heterozygous relatives and unrelated controls.

Methods. The genetic etiology was identified by whole genome sequencing and confirmed by Sanger sequencing. STK4 gene and protein expression was measured by quantitative RTPCR and immunoblotting, respectively. Cellular abnormalities were assessed by highthroughput RT-RCR, RNA-Seq, ELISA and polychromatic flow cytometry. Finally, antibody responses were delinated by ELISA and phage immunoprecipitation-sequencing.

Results. The affected patient exhibited partial loss of STK4 expression and complete loss of STK4 function. The patient suffered from recurrent viral and bacterial infections, most notably persistent Epstein-Barr virus viremia and pulmonary tuberculosis. Cellular and molecular analyses revealed abnormalities to the fractions of T-cell subsets, plasmacytoid dendritic cells, and NK cells. The transcriptional responses of the patient's whole blood and PBMC samples were reminiscent of dysregulated interferon signaling, impaired T immunity and increased Tcell apoptosis. Nonetheless, the patient had detectable vaccine-specific antibodies and IgG responses to various pathogens, consistent with a normal CD19+ B-cell fraction, albeit with a distinctive antibody repertoire, largely driven by herpesvirus antigens.

Conclusion. Patients with STK4 deficiency can exhibit broad impairment of immune function extending beyond lymphoid cells. 


\section{Keywords}

53 Human serine/threonine kinase 4 (STK4) deficiency; combined immunodeficiency; T cell 54 lymphopenia; interferon; antibody repertoire; transcriptomics 


\section{Introduction}

57 Human serine/threonine kinase 4 (STK4) deficiency is a rare autosomal recessive (AR) genetic disorder leading to a combined immunodeficiency with severe T cell lymphopenia. This condition is characterized by a predisposition to a wide range of bacterial and viral infectious diseases, mucocutaneous candidiasis, lymphomas and congenital heart disease (1). To date, STK4 deficiency has been reported in relatively few patients. Therefore, the extent to which immune signaling and host defense mechanisms are impaired or dysregulated in affected individuals remains incompletely understood. However, the spectrum of clinical manifestations associated with STK4 deficiency has been steadily increasing with each new case report.

STK4 deficiency was first reported by Nehme et al. in two patients from unrelated Turkish families harboring a homozygous nonsense mutation in the STK4 gene (2). The patients experienced complications due to recurrent bacterial and viral infections, most notably persistent Epstein-Barr virus (EBV) viremia, which ultimately resulted in Hodgkin B cell lymphoma. Due to weak expression of the homing receptors CCR7 and CD62L, the authors attributed the underlying mechanism of STK4 deficiency to increased death of naïve and proliferating $\mathrm{T}$ cells, and impaired homing of $\mathrm{CD} 8^{+} \mathrm{T}$ cells to secondary lymphoid organs (2). Abdollahpour et al. reported the cases of three siblings of Iranian descent with a homozygous premature stop codon in the STK4 gene (3). These patients suffered from T and B cell lymphopenia, intermittent neutropenia, and atrial septal defects, as well as recurrent bacterial and viral infections, mucocutaneous candidiasis, cutaneous warts, and skin abscesses. Interestingly, Schipp et al. reported a Turkish patient with STK4 deficiency who developed a highly malignant B cell lymphoma at the age of 10 years and a second, independent Hodgkin lymphoma 5 years later. However, no detectable EBV or other common virus infection was detected in this patient. The authors speculated that the lymphoma may have developed due

81 to the lack of the tumor suppressive function of STK4, or perturbed immune surveillance due 82 to the diminished $\mathrm{CD}^{+} \mathrm{T}$ cell compartment (4). In contrast, most malignancies reported in 
patients with STK4 deficiency are associated with prolonged EBV viremia, ultimately leading

84 to the development of B cell lymphomas (2, 5-7). More specifically, patients present with Hodgkin B cell lymphoma (2), extranodal marginal zone lymphoma of mucosa-associated lymphoid tissue (8), Burkitt's lymphoma (7), or maxillary sinus diffuse large B-cell lymphoma (9). Additional clinical features in patients with STK4 deficiency include salt-losing tubulopathy, suggestive of an acquired Gitelman syndrome, immune complex glomerulonephritis, and Castleman-like disease (10), juvenile idiopathic arthritis (11), human betapapillomavirusassociated epidermodysplasia verruciformis $(11,12)$ and primary cardiac T cell lymphoma (6).

Studies in mice and humans have shown that STK4 plays a pivotal role in lymphocyte function by regulating integrin-dependent $T$ lymphocyte trafficking, proliferation and differentiation (13, 14). Of note, the STK4 protein is broadly expressed in various human haemopoietic cells, most notably monocytes, and is not restricted to lymphocytes 96 (https://www.proteinatlas.org/ENSG00000101109-STK4). However, its role in T cellindependent functions is less well understood. Recently, Jørgensen et al. studied innate immune signaling in the context of STK4 deficiency by in vitro stimulation or infection of PBMCs obtained from an 11-year-old female STK4 ${ }^{-/-}$patient of a consanguineous Syrian family. These studies revealed defective type I/II and III interferon (IFN) responses to a variety of purified Toll-like receptor (TLR) agonists, live viruses or bacterial lysates due to impaired phosphorylation of the kinase TBK1 and the transcription factor IRF3 (15). The results also revealed increased apoptosis in STK4-deficient T cells and neutrophil granulocytes, as well as a decreased FoxO3a expression in STK4-deficient T cells, further supporting the important role of STK4 in T cell survival.

In this study, we identified an AR STK4 deficiency in a child from consanguineous parents, which was due to a novel homozygous stop-gain mutation in a region encoding a coiled-coil

109 domain located downstream of the kinase domain. We investigated the functional 110 consequences of the new variant on innate and adaptive cell-mediated, as well as humoral 
111 immune responses.

112 


\section{Methods}

114 Whole-genome sequencing

115 Genomic DNA (gDNA) was isolated from the peripheral blood of the subjects using DNeasy®

116 Blood \& Tissue Kits (Qiagen LLC, Germantown, MD, USA) according to the manufacturer's

117 instructions. Whole genome sequences were obtained using a standard library preparation

118 protocol and an Illumina HiSeq-X platform to generate 150-bp paired-end sequences. The raw

119 sequencing reads were mapped to the reference genome (GRCh37, hg19) using BWA

120 (version 0.7.15) (16), and genetic variants were called with the HaplotypeCaller in the GATK

121 suite (v.4.0) (17). The Variant Call Format (VCF) file, containing variants, was annotated using

122 SnpEff v.4.3 (18) and filtered for the candidate fitting the following criteria: (1) Being in the

123 coding region including the exonic, splice-site region, $(2)$ being rare $(<1 \%)$ in all mutation

124 databases (i.e., 1,000 genomes, gnomAD, ExAC, and ESP6500), and (3) co-segregates with

125 the phenotype in the family and follows a specific mode of inheritance (for example, AR). The

126 final list of variants was prioritized based on the literature search and whether any of the

127 associated genes had been linked to the patient's symptoms, such as known Mendelian genes

128 (https://omim.org).

129

130 Sanger sequencing. Primers seq_STK4_Ex8F (5'-GTCCGAAGCACAAAGAGAAAGA-3') 131 and seq_STK4_Ex8R (5'-CCAGCTCCAAGTTGATCCAATA-3') were designed to flank exon

1328 of the STK4 gene (NM_006282.5; LRG_535t1), checked for underlying single nucleotide

133 polymorphisms, and synthesized as described previously (19). The forward and reverse

134 primers were tailed with M13 sequences: CACGACGTTGTAAAACGAC (added to the 5' end

135 of the forward primer), and CAGGAAACAGCTATGACC (added to the 5' end of the reverse

136 primer). DNA was extracted from peripheral blood using a QIAsymphony® SP and

137 QIAsymphony DSP DNA Midi Kit (Qiagen Pty Ltd). DNA quantity and quality were assessed

138 using a NanoDrop® ND-1000 spectrophotometer (Thermo Scientific, Wilmington, DE, USA).

139 Genomic DNA (50ng) was subjected to conventional PCR using the following conditions: $95^{\circ} \mathrm{C}$ 
140 for 10 minutes, then 34 cycles of $95^{\circ} \mathrm{C}$ for 30 seconds, $60^{\circ} \mathrm{C}$ for 30 seconds and $72^{\circ} \mathrm{C}$ for 45

141 seconds, with a final incubation at $72^{\circ} \mathrm{C}$ for 5 minutes. Amplicons were checked for correct

142 size and non-specific amplification prior to ExoSAP-IT cleanup, cycle sequencing using

143 primers comprising the M13 tails, and BigDyeXTerminator cleanup followed by loading onto

144 an Applied Biosystems model 3500XL Genetic Analyzer. Sequence data were analyzed using

145 Applied Biosystems Sequence Analysis software, and Mutation Surveyor software.

147 RT-qPCR analysis of STK4 gene expression levels. For STK4 gene expression analysis,

148 PBMCs were isolated from the patient, parents and healthy donors by Ficoll-gradient

149 centrifugation. Cells were then incubated with T cell activation and expansion beads (anti150 CD2/CD3/CD28; Miltenyi Biotec, Gaithersburg, MD, USA) for 10 days in $5 \% \mathrm{CO}_{2}$ at $37^{\circ} \mathrm{C}$. On 151 day 3 , the medium was supplemented with $\mathrm{IL}-2(100 \mathrm{U} / \mathrm{ml})$ to promote cell proliferation. RNA 152 was extracted from 1 million cells using TRIzol lysis reagent (Thermo Fisher Scientific,

153 Carlsbad, CA, USA) according to the manufacturer's instructions. Briefly, 1 million cells were 154 lysed in $1 \mathrm{ml}$ of TRIzol for 3 minutes, then $140 \mu \mathrm{l}$ of chloroform were added, and the phases 155 were separated by centrifugation at $4^{\circ} \mathrm{C}$ and $16000 \times g$. RNA-containing liquid phase was 156 mixed to an equal volume of isopropanol and the mixture was incubated for 10 minutes at 157 room temperature for RNA precipitation. After centrifugation, precipitated RNAs were de158 salted by incubation with 1/10 volume of $5 \mathrm{M} \mathrm{NH} 4 \mathrm{OAc}$ and 2.5 volumes of $100 \%$ cold ethanol, 159 followed by washing with $75 \%$ ethanol and final elution in nuclease free water. The STK4 160 transcript was quantified using Fast SYBR Green Master Mix (Applied Biosciences) with the 161 following primers: forward 5'-TGGAGACGGTACAGCTGAGG-3'; reverse 5'162 ATAGCAACAATCTGGCCGGT-3'. All reactions were performed in triplicate using a 163 QuantStudio 6K Flex real-time PCR System (Applied Biosciences). Data were normalized to 164 the expression of the housekeeping gene RPLPO (Ribosomal protein, large, P0) and mean $165 \Delta \mathrm{Ct}^{-1}$ values were plotted. 
167 Western blot analysis. T cells were derived from PBMCs as described above and T cell

168 lysates (containing $20 \mu \mathrm{g}$ total protein) were separated using $4 \%-15 \%$ tris-glycine Bis-Tris

169 Gels (BioRad). Following electrophoresis, proteins were transferred to a polyvinylidene

170 fluoride (PVDF) membrane. After blocking with 5\% non-fat milk in tris-buffered saline (TBS)

171 for $1 \mathrm{~h}$, the membrane was incubated overnight at $4^{\circ} \mathrm{C}$ in TBS in the presence of anti-

172 recombinant STK4 antibody (diluted 1:5,000) (Abcam, clone EP1465Y, catalog no. ab51134),

$173 \alpha$-tubulin monoclonal antibody (diluted 1:10,000) (Cell Signaling Technology, Denvers, MA,

174 USA, catalog no. 3873), or anti- $\beta$-actin antibody (diluted 1:5,000) (Santa-Cruz Biotechnology,

175 Dallas, TX, USA, catalog no. sc-47778). Membranes were then washed $(3 \times 5 \mathrm{~min})$ with TBS

176 with $0.1 \%$ Tween-20 (TBS-T) and then incubated in TBS with a 1:5,000 dilution of either horse-

177 radish peroxidase (HRP)-conjugated goat anti-rabbit (Invitrogen, Frederick, MD, USA, catalog

178 no. 65-6120), or goat anti-mouse (Invitrogen, Frederick, MD, USA, catalog no. 31430)

179 secondary antibody, for $1 \mathrm{~h}$ at room temperature. Membranes were washed again $(3 \times 5 \mathrm{~min})$

180 in TBS-T. Immunoreactive proteins were visualized using enhanced chemiluminescence

181 (ECL $\left.{ }^{\mathrm{TM}}\right)$ (Amersham) according to the manufacturer's instructions. Images were acquired on

182 ChemiDoc MP imaging system (BioRad).

In vitro stimulation of whole blood and high-throughput gene expression analysis.

185 Peripheral blood was collected into sodium heparin vacutainer tubes. After incubation for $2 \mathrm{~h}$

186 at room temperature, $2 \mathrm{ml}$ WB was diluted with an equal amount of RPMI 1640 media

187 containing GlutaMax ${ }^{\mathrm{TM}}$ (Gibco). The samples were then stimulated for $2 \mathrm{~h}$ at $37^{\circ} \mathrm{C}$ with the

188 following agonists (final concentrations are shown): ultrapure LPS of E. coli K12 (InvivoGen)

189 at $10 \mathrm{ng} / \mathrm{ml}$; R848 (InvivoGen) at $3 \mu \mathrm{g} / \mathrm{ml}$; Poly(l:C) (high molecular weight) (InvivoGen) at 25

$190 \mu \mathrm{g} / \mathrm{ml}$; recombinant human IL-1 $($ R\&D Systems) at $20 \mathrm{ng} / \mathrm{ml}$; a 1:1 mix of recombinant human

191 IFN- $\alpha 2$ and IFN- $\beta$ (R\&D Systems) at 1,000 IU/ml each; recombinant human IFN-Y (R\&D

192 Systems) at $1,000 \mathrm{IU} / \mathrm{ml}$; recombinant human TNF-a (R\&D Systems) at $20 \mathrm{ng} / \mathrm{ml}$; 5'ppp-

193 dsRNA/LyoVec ${ }^{\text {TM }}$ (InvivoGen) at 1 $\mathrm{gg} / \mathrm{ml}$; Poly(dA:dT)/LyoVec ${ }^{\text {TM }}$ (InvivoGen) at $10 \mathrm{ng} / \mathrm{ml}$; 3'3'- 
194 cGAMP/LyoVec ${ }^{\text {TM }}$ (InvivoGen) at $10 \mu \mathrm{g} / \mathrm{ml}$; MDP (InvivoGen) at $10 \mathrm{ng} / \mathrm{ml}$; Dynabeads ${ }^{\mathrm{TM}}$

195 Human T-Activator CD3/CD28 (Invitrogen) at 400,000 beads/ml; AffiniPure $F\left(a^{\prime}\right)_{2}$ fragment

196 goat anti-human $\lg \mathrm{G}+\lg \mathrm{M}(\mathrm{H}+\mathrm{L})$ (Jackson ImmunoResearch) at $10 \mu \mathrm{g} / \mathrm{ml}$; or PMA/ionomycin

197 calcium salt (Sigma) at $40 \mathrm{ng} / \mathrm{ml}$ and $1 \mu \mathrm{g} / \mathrm{ml}$. Similarly, cells were incubated for $2 \mathrm{~h}$ at $37^{\circ} \mathrm{C}$ 198 with LyoVec $^{\mathrm{TM}}$ alone or RPMI media as unstimulated control samples. Subsequently, three 199 volumes of Tempus ${ }^{\mathrm{TM}}$ solution (Applied Biosystems) were added for cell lysis and RNA 200 stabilization before total RNA was isolated using the Tempus ${ }^{\text {TM }}$ Spin RNA isolation kit (Applied 201 Biosystems). RNA quality and quantity were assessed using an Agilent 2100 Bioanalyzer 202 (Agilent Technologies) and NanoDrop 1000 (Thermo Fisher Scientific). The cDNA of all 180 203 target genes and six housekeeping genes was generated from $40 \mathrm{ng}$ total RNA using a reverse 204 transcription master mix (Fluidigm) and quantified using custom Delta Gene ${ }^{\mathrm{TM}}$ assays in combination with 96.96 Dynamic Array ${ }^{\mathrm{TM}}$ IFCs and the BioMark ${ }^{\mathrm{TM}}$ HD microfluidic system 206 (Fluidigm) in accordance with the manufacturer's instructions. All 180 target genes and six 207 housekeeping genes (Supplemental Table S3) were selected a priori from a larger set of 2081,088 transcripts, represented by 66 sets of co-expressed genes (transcriptional modules), 209 which had previously been found to be responsive in WB stimulation with purified PRR 210 agonists, recombinant cytokines and pyogenic bacteria (20). We selected three representative 211 genes per transcriptional module for a total of 60 modules based on their closeness to the 212 median absolute expression of all transcripts assigned to a given module. Six modules were 213 excluded from the analysis due to limited functional annotation. Housekeeping genes were 214 selected from the same dataset (GSE25742) as previously described (21). Expression levels 215 of the 180 target genes were quantified relative to the six housekeeping genes using the $2^{-}$ $216 \Delta^{\Delta \Delta C t}$ method (22). We evaluated fold changes in expression as $\log _{2}$-transformed expression 217 level (log2FC) for each target gene under each stimulation condition and filtered for genes with 218 a $|\log 2 \mathrm{FC}| \geq 1$ in at least two samples. Next, we computed z-score values of the log2FC for 219 each gene and condition in the patient using two unrelated control subjects and the three 220 heterozygous family members as controls. Finally, we used principal component analysis to 221 estimate the variance in z-scores across samples. The first two principal components 
described approximately $75 \%$ of the variance in the z-score profile of samples and a distinct segregation of patient and control subjects was observed (Supplemental Figure S3). We extracted the contribution factor for each gene from the PCA and filtered for pairs of target gene and stimulation condition that showed a variance of $|\log 2 \mathrm{FC}|>1$ between the patient and control subjects, resulting in the identification of 28 target genes that were differentially expressed under one or more (i.e. up to eight) in vitro stimulation conditions (Figure 4 and

\section{Supplemental Table S3)}

PBMC stimulation and RNA-Seq analysis. Peripheral blood was collected into sodium heparin vacutainer tubes, PBMCs were isolated by Ficoll-Paque (GE Healthcare) density gradient centrifugation according to the manufacturer's instructions and frozen in fetal bovine serum containing $20 \%$ dimethyl sulfoxide. For in vitro stimulation, PBMCs where thawed, rested for $24 \mathrm{~h}$ and then stimulated with a $1: 1 \mathrm{mix}$ of recombinant human IFN- $\alpha 2$ and IFN- $\beta$ (R\&D Systems), PMA/ionomycin, or left unstimulated as described for the stimulation of whole blood samples. Total RNA was isolated using the RNeasy Mini Kit (QIAGEN) according to the manufacturer's instructions. RNA integrity and purity were evaluated using a Bioanalyzer 2100

238 (Agilent Technologies Genomics). cDNA was generated using the SMARTer v4 Ultra ${ }^{\circledR}$ Low Input RNA for Sequencing Kit (Takara Bio). Resulting cDNA was quantified and size-controlled using a Bioanalyzer 2100 (Agilent Technologies Genomics). cDNA was normalized to $1 \mathrm{ng} / \mu \mathrm{l}$ and libraries were prepared using the Nextera XT DNA Library Preparation Kit (Illumina) and

242 the Nextera XT Index Kit v2 set A (Illumina), respectively, in accordance with the manufacturer's instructions. 150 bp paired-end sequencing to approximately 20 million reads per sample was performed using a HiSeq4000 system (Illumina). Raw reads were aligned to the UCSC human genome assembly version hg38 using STAR aligner (23). The HTSeq-count

246 (24) tool was used to count the number of reads that mapped to each gene feature. Read counts were adjusted to mitigate batch effects by negative binomial regression models using comBat-Seq (25). After read counts were normalized, we removed genes expressed at low 
levels using the HTSFilter and the default protocol. We finally calculated log2FC values for

250 each subject and stimulation condition relative to the unstimulated condition of the same

251 subject using edgeR (26). Genes with a $|\log 2 \mathrm{FC}| \geq 1$ were considered differentially expressed.

252 Residual responses were calculated as described previously (27). Gene enrichment analysis

253 was performed using the QIAGEN IPA software application.

Phage immunoprecipitation-sequencing (PhIP-Seq). The VirScan phage library used for PhIP-Seq in the present study was obtained from S. Elledge (Brigham and Women's Hospital and Harvard Medical School, Boston, MA, USA). PhIP-Seq was performed as described previously (28-30) using an expanded version of the original VirScan phage library (31). We computed species-specific adjusted score values as described earlier $(30,32)$. Pooled human plasma used for IVIg (Privigen® CSL Behring AG) and human IgG-depleted serum (Molecular Innovations, Inc.) served as additional controls.

Flow cytometry. Lymphocyte subsets were assessed using Duraclone assays (Beckman Coulter) to delineate major lymphocyte subsets (IM Phenotyping Basic) and more specifically, $\mathrm{T}$ cell and dendritic cell subsets (IM T cell and IM DCs) according to the manufacturer's protocol. Samples were analyzed with a FACSymphony A5 Flow Cytometer (BD Biosciences) and FlowJo 10.5.2 software.

Absolute blood cell counts, total immunoglobulin levels, vaccine-specific antibody measurements and QuantiFERON-TB Gold ELISA

271 Complete blood cell and lymphocyte counts, total immunoglobulin measurements (i.e., IgG, $272 \lg A, \lg M$ ), vaccine-specific antibody measurements (i.e., anti-diphtheria toxoid IgG, anti273 Haemophilus influenzae Type B, anti-pneumococcus capsular poly Ab, anti-tetanus toxoid 274 antibody) and whole blood IFN- $\gamma$ measurements in response to Mtb peptide antigens 275 (QuantiFERON-TB Gold ELISA, QIAGEN, Germantown, MD, USA) were carried out and 276 interpreted by laboratories accredited by the College of American Pathologists (CAP) following 
277 the manufacturer's instructions and clinical guidelines. Normal ranges were provided in tables 278 and figures as appropriate. An "indeterminate" test result of the QuantiFERON-TB Gold ELISA 279 indicated the patient's lymphocytes also did not respond to mitogen stimulation (can be seen 280 in immunodeficiency, lymphopenia, overwhelming infection, malnutrition, and with 281 immunosuppressive medications).

282

283 Statistical analysis. For PhIP-Seq experiments, we imputed - $\log _{10}(P$-values $)$ by fitting a zero284 inflated generalized Poisson model to the distribution of output counts followed by regression 285 of the parameters for each peptide sequence based on the input read count. Peptides that 286 passed a reproducibility threshold of $-\log _{10}(P$-value $) \geq 2.3$ in two technical sample replicates 287 were considered significantly enriched. 


\section{Results}

290 Clinical description of the case and family members. The patient was the third child of

291 consanguineous parents (first-degree cousins). The patient had a younger sibling who was

292 healthy, as were both parents. Of note, the parents reported the death of the two elder siblings

293 of the patient. One died with a history of chronic headache, coughing and Iymphoma, while

294 the other died with a history of chronic coughing (Figure 1A). However, no detailed medical

295 records or genetic data were available for the two deceased siblings.

297 The patient suffered from recurrent skin rashes starting from infancy, recurrent chest infections

298 since early childhood, and an overall failure to thrive with low weight gain and short stature

299 (data not shown). The patient's early medical history included a productive cough of 300 yellow/white mucoid sputum associated with on/off fever, which was more common at bedtime;

301 however, early medical records were very limited and likely to be incomplete. The patient was

302 more closely followed clinically starting at elementary school age. He was diagnosed with

303 bronchiectasis and asthma, and started on asthma control medication, including Ventolin. He

304 also experienced complications due to recurrent viral and bacterial infections, chronic

305 suppurative otitis media and recurrent pneumonia. The patient was also diagnosed with

306 tuberculosis (TB), which was confirmed by Mycobacteria tuberculosis complex positive culture,

307 while a QuantiFERON assay, performed in parallel, had an "indeterminate" test result (see

308 methods for details). The patient was subsequently treated for pulmonary TB for approximately

309 one year. After being off treatment for another year, the patient suffered from TB reactivation,

310 and was again put on anti-TB medication (cycloserine, linezolid, moxifloxacin and

311 pyrazinamide) for two years. He also presented with a lower chest infection as a teenager and

312 a chest X-ray confirmed lower left consolidation. A sputum culture revealed heavy growth of

313 Haemophilus influenzae and Streptococcus pneumoniae, as well as light growth of methicillin-

314 resistant Staphylococcus aureus. About a year later, the patient was hospitalized again with a

315 second episode of lower chest infection by $H$. influenzae and multiple-drug resistant Klebsiella 
316 pneumoniae. EBV viremia was detected during early teenage years, and repeated testing

317 confirmed persistent EBV viremia up to the most recent follow-up. During the teenage years,

318 he also suffered from intermittent neutropenia and severe lymphopenia (Supplemental

319 Figures S1A, S1B and S1C) with markedly decreased naïve CD45RA ${ }^{+}$cells $(11.1 \%$; normal

320 range: $46 \%-77 \%$ ); the onset may have been earlier but was not detected due to the late

321 diagnosis. Antibody responses to childhood vaccination were within the normal range

322 (Supplemental Table S1).

323

324 Homozygosity for a stop-gain mutation in the STK4 gene. We performed whole genome

325 sequencing on the patient and all of his immediate family members, except the deceased

326 sisters. The analysis revealed a rare, homozygous nonsense mutation in the STK4 gene

327 (NM_006282.2:c.871C>T, p.Arg291*) in the patient, whereas both parents and the younger

328 sibling were identified as heterozygous carriers, suggesting an AR inheritance pattern (Figure

329 1A). The STK4 genotypes of the patient and his family members were confirmed by clinical

330 Sanger sequencing (Figure 1B). The combined annotation-dependent depletion (CADD)

331 score of the variant was 42, providing further evidence of its deleteriousness (Figure 1C and

332 D).

334 The mutant STK4 allele is a loss-of-function (LOF) variant. The mutant STK4 protein was

335 not detected in PBMC-derived T cells from the patient by Western blot analysis using a

336 monoclonal antibody directed against the $\mathrm{N}$-terminus of the protein, whereas intermediary

337 levels of the STK4 protein were found in the parents compared to two unrelated healthy

338 controls (Figure 1E). We also assessed the impact of the STK4 variant at the transcript level.

339 A STK4 transcript was detectable in PBMC-derived T cells of the patient, albeit at reduced

340 levels compared to controls with a wild-type genotype and the heterozygous parents

341 (Supplemental Figure S2A and B). 
350 population was not affected in the patient $(11.7 \%)$ when compared to the controls (range

$351 \quad 10.9 \%-20.8 \%$ ) (Figure 2A). Similarly, CD56 ${ }^{+}$CD3 $^{-}$NK cells of the patient (4.59\%) were within

352 the normal range compared to the control subjects (range 1.87\%-6.82\%) (Figure 2B).

353 However, we noticed a significant increase of the CD56 bright $N K$ cell subset in the patient $354(1.78 \%)$ compared to all controls (range $0.058 \%-0.42 \%$ ) (Figure 2B). We then assessed $355 \mathrm{CD}^{+} \mathrm{T}$ cell subsets and found an increase in programmed death-1 (PD-1)-expressing T helper 356 cells in the patient (Figure 2D). Next, we analyzed PBMCs expression of CD45RA and CCR7 357 and detected low frequencies of $C D 45 R A^{+} C C R 7^{+}$double-positive naïve T cells in the patient, 358 while his CD45RA'CCR7- effector memory population was increased (Figure 2E). Similarly,

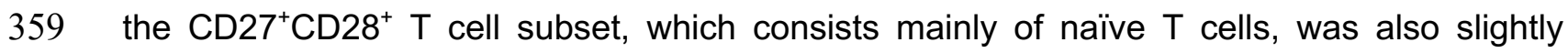
360 decreased in the patient (Figure 2F). Finally, assessment of the dendritic cell (DC) subsets 361 showed a decrease of $\mathrm{CD} 11 \mathrm{c}^{-} \mathrm{CD} 123^{+}$plasmacytoid $\mathrm{DCs}$ (pDCs) in the patient, while his

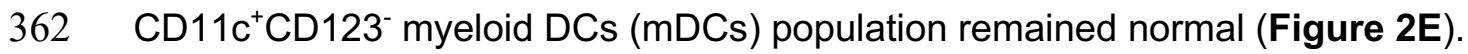

364 The patient has a distinct antiviral antibody repertoire. To further assess the humoral 365 immunity status of the patient, we performed large-scale antibody profiling by phage 366 immunoprecipitation-sequencing (PhIP-Seq) using serum samples obtained from the patient. 367 We found the patient to be seropositive for antibodies specific to a variety of common viruses 368 and bacteria, including human herpesviruses (HHV)-4 (EBV), -5 (CMV) and -8 , enterovirus 369 (EV)-B, and human respiratory syncytial virus (HRSV), human rhinoviruses (HRV) A and B, S. 370 pneumoniae and S. aureus (Figure 3A). The antibody repertoire breadth in the patient was 
371 similar to that of the controls (Figure 3B). Nevertheless, principal component analysis of the

372 enriched antibody-antigen interactions showed an overall distinct antibody repertoire in the

373 patient compared to those of his family members and unrelated controls (Figure $3 \mathbf{C}$ ), which

374 was largely driven by antibodies targeting HHV-4 and -5 antigens (Figure 3D).

Gene expression signatures suggest dysregulated interferon signaling and impaired T cell activation, inhibition of $T$ cell proliferation and increased $T$ cell apoptosis. To further elucidate the functional consequences of STK4 deficiency at the molecular level, we performed gene expression analyses using either whole blood (WB) samples or PBMCs isolated from the patient and control subjects following in vitro stimulation with a variety of immune activators. First, we stimulated WB of the patient, his family members and an unrelated control subject with purified pattern recognition receptor (PRR) agonists [lipopolysaccharides of E. coli K12 (LPS 12 ) (a TLR4 agonist), muramyl dipeptide (MDP) (a NOD2 agonist), Poly(I:C) (a TLR3 agonist), Poly(dA:dT) (a multiple-PRR agonist), resiquimod (R848) (a TLR7/8 agonist), cyclic guanosine monophosphate-adenosine monophosphate (cGAMP) (a STING agonist), and 5' triphosphate double-stranded RNA (5'ppp-dsRNA) (a RIG-

387 I agonist)], cytokines [IFN- $\alpha /$ IFN- $\beta$ (an interferon- $\alpha / \beta$ receptor IFNAR agonist), IFN- $\gamma$, IL- $1 \beta$ and

388 TNF- $\alpha$ ], a potent mitogen [phorbol 12-myristate 13-acetate (PMA)/ionomycin] and BCR or 389 TCR activators. We measured the expression of 180 functionally well-annotated target genes, 390 which we had selected a priori from a larger set of genes responsive to WB stimulation with 391 purified PRR agonists, recombinant cytokines and pyogenic bacteria (20) (see methods and 392 Supplemental Table S3 for further details); mock stimulations served as controls. We then 393 filtered for differentially expressed genes (DEGs) among the 180 target genes for which the 394 transcriptional responses in the patient's WB samples to any of the in vitro stimulation 395 conditions showed high variance in comparison to those of the other family members and the 396 unrelated control (Supplemental Figure S3). The identified DEGs were associated with 397 caspases and apoptosis, type I and II interferon signaling, inflammation, cell signaling, and 
ubiquitination, as well as cell movement and phagocytosis (Figure 4 and Supplemental Table

399 S4).

400 Finally, we performed mRNA-Seq of PBMCs isolated from the patient and following stimulation 401 with PMA/ionomycin, IFN- $\alpha / \mathrm{IFN}-\beta$, or unstimulated. Both parents and three unrelated, healthy 402 controls were assessed for comparison. We filtered for DEGs in response to each stimulation 403 condition and found a profound dysregulation of IFN-regulated gene expression, although IFN404 induced gene expression was not completely abrogated (Figure 5A). We then performed a 405 gene enrichment analysis on the identified DEGs using the QIAGEN Ingenuity Pathway 406 Analysis (IPA) software application. We focused our downstream analysis on canonical 407 pathways that were differentially activated or inhibited in the patient's cells, compared to those 408 of the controls. In response to IFNAR activation, nine pathways were differentially regulated in 409 the patient compared to the controls, most of which are linked to T cell signaling and apoptosis, 410 cell proliferation, oxidative stress and interestingly, IL-23 signaling (Figure 5B). Similarly, we 411 observed several pathways that are normally repressed following mitogen activation, but 412 instead were highly activated or dysregulated in the patient's cells. These included pathways 413 primarily involved in $\mathrm{T}$ cell effector functions, $\mathrm{T}$ and $\mathrm{B}$ cell activation, cell cycle arrest and 414 apoptosis (Figure 5B). Given the apparent dysregulation of IL-23 signaling in the patient's 415 cells, we also examined absolute IL-23 and IFNG gene expression, either at baseline, or 416 following stimulation with PMA/ionomycin. We found that in comparison to the control subjects, 417 IL-23 gene expression in the patient was highly impaired at baseline and largely unresponsive 418 to PMA/ionomycin stimulation, whereas IFNG gene expression appeared normal 419 (Supplemental Figure S3C and D). 


\section{Discussion}

421 Due to the relatively small number of patients reported to date, the extent to which immune

422 signaling and host defense mechanisms are impaired or dysregulated in patients with STK4

423 deficiency remains incompletely understood. Here, we describe a male pediatric patient with

424 AR complete STK4 deficiency and a clinical history of recurrent viral and bacterial infections,

425 persistent EBV viremia and pulmonary TB. The underlying stop-gain mutation was found to be

426 located in a genomic region that encodes the coiled-coil domain of STK4, downstream of its

427 protein kinase domain. We were unable to detect even a truncated STK4 protein in the patient

428 using a monoclonal antibody to the N-terminal region of STK4, suggesting that protein

429 expression of the mutated allele is completely abrogated due to nonsense-mediated decay.

431 In accordance with earlier case reports $(5,8,33)$, we found that the PBMCs isolated from the 432 patient in this study had reduced fractions of $\mathrm{CD}^{+}$naïve but increased effector memory $\mathrm{T}$ 433 helper cell subsets compared with those in the STK $4^{\text {wt/mut }}$ family members and an unrelated

434 STK $4^{w t / w t}$ control. Furthermore, flow cytometric analysis showed a considerable proportion of 435 the remaining $T$ helper cell subset in the patient expressed higher levels of PD-1, and our 436 RNA-Seq analyses revealed dysregulation of several pathways in the patient, suggesting 437 elevated $\mathrm{T}$ cell exhaustion and impaired effector functions of the residual $\mathrm{T}$ cells. Whether this 438 is a consequence of persistent EBV viremia (34-36) or an intrinsic feature of STK4 deficiency, 439 or perhaps both, remains to be established. Previous studies have shown that EBV 440 reactivation correlates with the expression of PD-1/PD-L1 antigens in patients with proliferative 441 glomerulonephritis (37). On the other hand, $\mathrm{CD}^{+} \mathrm{T}$ cell lymphopenia has also been reported 442 in STK4-deficient patients in the absence of detectable EBV infection (4). In addition, the 443 patient presented with episodes of intermittent neutropenia, which is consistent with previous 444 observations $(2,3,6,9)$. 
446 Our results also highlight that STK4 deficiency can lead to the impairment of a variety of T cell-

447 independent and innate immune responses. Indeed, we detected a considerable proportion of 448 CD56 ${ }^{\text {bright }}$ NK cells in the PBMCs isolated from the patient. While these cells constitute only a 449 small fraction of NK cells in the peripheral blood in healthy individuals, they represent the 450 majority of NK cells in secondary lymphoid tissues. CD56 ${ }^{\text {bright }}$ NK cells are thought to be the 451 precursors of NK cells (38) and may have immunoregulatory properties (39). We also 452 observed a decreased fraction of pDCs in the patient's peripheral blood. Whether this is an 453 indirect consequence of active EBV infection, as shown in mouse studies (40), or whether low 454 levels of pDCs contribute directly to a lack of EBV control, remains unclear. In accordance with 455 the recent findings reported by Jørgensen et al. (15), we observed dysregulated type I and II 456 IFN signaling in the patient's cells. Interestingly, transcriptomic analysis of the patient's PBMCs 457 in response to IFNAR activation in vitro revealed a profound dysregulation of IFN-regulated gene expression, affecting interferon-stimulated genes (ISGs) that are primarily associated with $\mathrm{T}$ cell activation and proliferation, although IFN-induced gene expression was not 460 completely abrogated. This suggests that suboptimal IFN signaling may contribute to the T 461 cell immunodeficiency and the vulnerability of STK4-deficient patients to viral infection and 462 cancer development. However, overall fractions of $\mathrm{CD}_{19^{+}} \mathrm{B}$ cells (Figure 2A) and $\lg$ antibody responses to childhood vaccination (Supplemental Table S1) or common microbial infection (Figure 3) did not appear to be diminished in our patient, apart from our observation that antibody specificities were largely targeting HHV-4 and -5 antigens. Of note, a recent study in STK $4^{-/}$mice and nine patients from five unrelated families with STK4 deficiency has

467 suggested that STK4 is required for normal humoral immunity, since KO mice and patients 468 had reduced marginal zone $B(M Z B)$ cells, as well as reduced numbers of innate-like $B-1 b$ cell 469 subsets, while the overall fractions of circulating CD19+ $\mathrm{B}$ cells were normal as in our patient 470 (41). This raises the question whether patients with STK4 deficiency may also be selectively 471 impaired to mount robust T cell-independent, polysaccharide-specific antibody responses to 472 control natural infection with encapsulated bacteria, such as $H$. influenzae, K. pneumoniae 473 and S. pneumoniae, which would be consistent with the clinical history of our patient. 
474 Polysaccharide-specific antibody responses (or the lack thereof) would have been 475 undetectable by the PhIP-Seq assay performed in this study, as it exclusively detects 476 antibodies that target protein antigens and has limitations for the detection of conformational 477 as well as post-translationally modified epitopes (28). High efficacy of plain polysaccharide478 based vaccines also depends on the maturation of MZB cells, which usually does not occur 479 until the second year of life (42). In our patient, the specific antibody responses were at the 480 lower end of our laboratory reference range (Supplemental Table S1). However, anti481 pneumococcal polysaccharide antibodies cannot necessarily be utilized as markers of MZB 482 cell-mediated immunity, due to the introduction of the conjugate pneumococcal vaccine into 483 the local routine immunization schedule. The literature shows variability for specific antibody 484 responses in STK4-deficient patients, ranging from normal to absent (41). The infection history 485 of our patient with H. influenzae, K. pneumoniae and S. pneumoniae could have also interfered with the utility and interpretation of vaccine response tests using plain polysaccharide

487 vaccines. Therefore, humoral immunity of patients with STK4 deficiency towards encapsulated 488 bacteria requires further investigation.

We also demonstrated a profound impairment of $I L-23$ gene expression in the patient's PBMCs, both at baseline and following in vitro stimulation. IL-23 is produced by innate lymphoid cells, gamma-delta T cells, DCs and macrophages, and it has been shown that IL23-dependent IFN- $\gamma$ immunity plays a pivotal role in controlling Mycobacterium tuberculosis (Mtb) infection (43). It is therefore tempting to speculate that impaired IL-23 gene expression may have contributed to the susceptibility of the patient to pulmonary TB, confirmed on sputum

496 culture. Despite the clinical evidence of pulmonary TB, the patient's QuantiFERON test result 497 was indeterminate, i.e., the patient's lymphocytes did not respond to mitogen stimulation. This 498 is likely to reflect a combination of the cellular dysfunction and profound lymphopenia. Of note, 499 Radwan et al. (7) also speculated that complications in a 9-year-old Egyptian boy with STK4 deficiency were associated with mycobacterial infection, albeit tuberculin skin-test results were 
501 negative and the results from QuantiFERON tests were also inconclusive in this Egyptian

502 patient.

503

504 It remains unclear whether malignancies in STK4-deficient patients are a secondary consequence of persistent EBV viremia, or whether patients with STK4 deficiency are inherently prone to malignancies due to dysregulation of oncogenes even in the absence of

507 EBV infection (4). Interestingly, our RNA-Seq experiments revealed upregulation of mitogen-

508 induced B cell-activating factor (BAFF) receptor gene (TNFRSF13C) expression in the patient,

509 suggesting activation of BAFF signaling, in contrast to the controls where this pathway was

510 inhibited following PMA/ionomycin stimulation (Figure 5 and Supplemental Table S2).

511 Studies in vitro and in mice have shown that EBV drives autonomous B cell proliferation (44),

512 which also depends on T cell-independent survival signals provided by the BAFF receptor.

513 Excessive BAFF levels have been implicated in several B-lineage malignancies (45-48), which

514 have also been reported in the context of STK4 deficiency with or without EBV viremia $(2,5-$

$5158,10)$. Our observations provide further mechanistic insights into the susceptibility of STK4-

516 deficient patients to malignancies, although they do not allow firm conclusions about the role

517 of EBV in this process. Nonetheless, it is tempting to speculate that STK4-deficient patients,

518 particularly those with persistent EBV viremia, may benefit from treatment with immune

519 checkpoint inhibitors. Using a humanized mouse model, Ma et al. (49) demonstrated a direct

520 beneficial effect of PD-1/CTLA-4 blockade mediated by monoclonal antibodies against PD-1

521 or CTLA-4 alone, or in combination, on EBV-associated B cell lymphomas, thereby providing

522 further evidence in support of this hypothesis. On the other hand, several research groups

523 have reported TB reactivation or primary Mtb infections in cancer patients who received

524 checkpoint inhibitors (50-52). Therefore, the potential benefit of the use of checkpoint inhibitors

525 in patients with STK4 deficiency requires further investigation. 


\section{Declarations}

528 Funding: This work was supported by a grant from the Qatar National Research Fund (Grant no. NPRP9-251-3-045) and funds from Sidra Medicine (SDR400048, SDR400013).

Conflicts of interest/Competing interests: The authors have no conflicts of interest to declare that are relevant to the content of this article.

Availability of data and material: All processed data are available in the manuscript or the supplementary materials. Raw and processed sequence read data from the PhIP-Seq experiments are available from the corresponding author on reasonable request. Raw and processed RNA-Seq data are available at the Gene Expression Omnibus (GEO), series accession number GSE166761.

Code availability: Python in-house scripts used in this study are available from the corresponding author on reasonable request.

543 Authors' contributions: NM conceived the study and supervised the project. AG, SB, RM,

544 BL, MR, MA, FA, DP and KD designed and performed experiments. TK, AG, SB, WA, TH and 545 NM analyzed and interpreted the flow cytometry, PhIP-Seq and high-throughput gene expression data as well as the RNA-Seq data. RM and BL analyzed STK4 gene and protein expression. KF and RC provided and analyzed the WGS data. MJ, DL, MK, AH and MA

548 contributed the clinical data. AS, QZ, ECS, FA, MR and AR helped with the subject recruitment. AG, NM and SB wrote the paper.

551 Ethics approval: The study protocol was approved by the institutional review board (IRB) of 552 Sidra Medicine (protocol no. 1601002512) and Weill Cornell Medicine, Qatar (protocol no. 13553 00065). 
555 Consent to participate: Written informed consent was obtained from each participant in 556 accordance with local regulations governing human subject research.

558 Consent for publication: All authors have seen and approved the manuscript, which has not 559 been accepted or published elsewhere.

\section{Acknowledgments}

563 The authors would like to thank the patient and his family for participating in this study. The 564 authors also thank Stephen Elledge (Brigham and Women's Hospital, Harvard University 565 Medical School) for kindly providing the VirScan phage library, the Integrated Genomics Core 566 and Deep Phenotyping Services team of Sidra Medicine for their assistance with the high567 throughput PCR experiments, RNA sequencing and flow cytometry; and Jessica Tamanini 568 (Insight Editing London) for proofreading and editing the manuscript. 


\section{References}

571 1. Tangye SG, Al-Herz W, Bousfiha A, Chatila T, Cunningham-Rundles C, Etzioni A, et al. 572 Correction to: Human Inborn Errors of Immunity: 2019 Update on the Classification from 573 the International Union of Immunological Societies Expert Committee. J Clin Immunol. 2020;40(1):65.

575

2. Nehme NT, Schmid JP, Debeurme F, Andre-Schmutz I, Lim A, Nitschke P, et al. MST1 mutations in autosomal recessive primary immunodeficiency characterized by defective naive T-cell survival. Blood. 2012;119(15):3458-68.

3. Abdollahpour H, Appaswamy G, Kotlarz D, Diestelhorst J, Beier R, Schaffer AA, et al. The phenotype of human STK4 deficiency. Blood. 2012;119(15):3450-7.

4. Schipp C, Schlutermann D, Honscheid A, Nabhani S, Holl J, Oommen PT, et al. EBV Negative Lymphoma and Autoimmune Lymphoproliferative Syndrome Like Phenotype Extend the Clinical Spectrum of Primary Immunodeficiency Caused by STK4 Deficiency. Front Immunol. 2018;9:2400.

5. Dang TS, Willet JD, Griffin HR, Morgan NV, O'Boyle G, Arkwright PD, et al. Defective Leukocyte Adhesion and Chemotaxis Contributes to Combined Immunodeficiency in Humans with Autosomal Recessive MST1 Deficiency. J Clin Immunol. 2016;36(2):11722.

6. Sherkat R, Sabri MR, Dehghan B, Bigdelian H, Reisi N, Afsharmoghadam N, et al. EBV lymphoproliferative-associated disease and primary cardiac T-cell lymphoma in a STK4 deficient patient: A case report. Medicine (Baltimore). 2017;96(48):e8852.

7. Radwan N, El-Owaidy R, El-Sayed ZA, Abdel-Baky A, El-Haddad A, Rashad H, et al. A Case of STK4 Deficiency with Complications Evoking Mycobacterial Infection. J Clin Immunol. 2020;40(4):665-9. 
594 8. Lum SH, Bonney D, Cheesman E, Wrignt NB, Hughes S, Wynn R. Successful Curative 595 Therapy With Rituximab and Allogeneic Haematopoietic Stem Cell Transplantation for 596 MALT Lymphoma Associated With STK4-Mutated CD4+ Lymphocytopenia. Pediatr $597 \quad$ Blood Cancer. 2016;63(9):1657-9.

9. Ashrafi F, Klein C, Poorpooneh M, Sherkat R, Khoshnevisan R. A case report of sinusoidal diffuse large B-cell lymphoma in a STK4 deficient patient. Medicine

10. Al-Saud B, Alajlan H, Sabar H, Anwar S, Alruwaili H, Al-Hussain T, et al. STK4 Deficiency in a Patient with Immune Complex Glomerulonephritis, Salt-Losing Tubulopathy, and Castleman's-Like Disease. J Clin Immunol. 2019;39(8):823-6.

11. Sharafian S, Ziaee V, Shahrooei M, Ahadi M, Parvaneh N. A Novel STK4 Mutation Presenting with Juvenile Idiopathic Arthritis and Epidermodysplasia Verruciformis. J Clin

12. Crequer A, Picard C, Patin E, D'Amico A, Abhyankar A, Munzer M, et al. Inherited MST1 Immunol. 2019;39(1):11-4.

13. Choi J, Oh S, Lee D, Oh HJ, Park JY, Lee SB, et al. Mst1-FoxO signaling protects Naive T lymphocytes from cellular oxidative stress in mice. PLoS One. 2009;4(11):e8011.

14. Ueda Y, Kondo N, Kinashi T. MST1/2 Balance Immune Activation and Tolerance by Orchestrating Adhesion, Transcription, and Organelle Dynamics in Lymphocytes. Front Immunol. 2020;11:733.

15. Jorgensen SE, Al-Mousawi A, Assing K, Hartling U, Grosen D, Fisker N, et al. STK4 Deficiency Impairs Innate Immunity and Interferon Production Through Negative Regulation of TBK1-IRF3 Signaling. J Clin Immunol. 2020. 
16. Li H, Durbin R. Fast and accurate short read alignment with Burrows-Wheeler transform. Bioinformatics. 2009;25(14):1754-60.

17. DePristo MA, Banks E, Poplin R, Garimella KV, Maguire JR, Hartl C, et al. A framework for variation discovery and genotyping using next-generation DNA sequencing data. Nat Genet. 2011;43(5):491-8.

18. Cingolani $P$, Platts $A$, Wang le $L$, Coon $M$, Nguyen $T$, Wang $L$, et al. A program for annotating and predicting the effects of single nucleotide polymorphisms, SnpEff: SNPs in the genome of Drosophila melanogaster strain w1118; iso-2; iso-3. Fly (Austin). 2012;6(2):80-92.

19. Love JM, Prosser D, Love DR, Chintakindi KP, Dalal AB, Aggarwal S. A novel glycine decarboxylase gene mutation in an Indian family with nonketotic hyperglycinemia. $\mathrm{J}$ Child Neurol. 2014;29(1):122-7.

20. Alsina L, Israelsson E, Altman MC, Dang KK, Ghandil P, Israel L, et al. A narrow repertoire of transcriptional modules responsive to pyogenic bacteria is impaired in patients carrying loss-of-function mutations in MYD88 or IRAK4. Nat Immunol. 2014;15(12):1134-42.

21. de Jonge HJ, Fehrmann RS, de Bont ES, Hofstra RM, Gerbens F, Kamps WA, et al. Evidence based selection of housekeeping genes. PLoS One. 2007;2(9):e898.

22. Livak KJ, Schmittgen TD. Analysis of relative gene expression data using real-time quantitative PCR and the 2(-Delta Delta C(T)) Method. Methods. 2001;25(4):402-8.

23. Dobin A, Davis CA, Schlesinger F, Drenkow J, Zaleski C, Jha S, et al. STAR: ultrafast universal RNA-seq aligner. Bioinformatics. 2013;29(1):15-21.

24. Anders S, Pyl PT, Huber W. HTSeq--a Python framework to work with high-throughput sequencing data. Bioinformatics. 2015;31(2):166-9. 
25. Zhang Y, Parmigiani G, Johnson WE. ComBat-seq: batch effect adjustment for RNA-seq count data. NAR Genom Bioinform. 2020;2(3):Iqaa078.

26. Robinson MD, McCarthy DJ, Smyth GK. edgeR: a Bioconductor package for differential expression analysis of digital gene expression data. Bioinformatics. 2010;26(1):139-40.

27. Li J, Ritelli M, Ma CS, Rao G, Habib T, Corvilain E, et al. Chronic mucocutaneous candidiasis and connective tissue disorder in humans with impaired JNK1-dependent responses to IL-17A/F and TGF-beta. Sci Immunol. 2019;4(41).

28. Mohan D, Wansley DL, Sie BM, Noon MS, Baer AN, Laserson U, et al. Publisher Correction: PhIP-Seq characterization of serum antibodies using oligonucleotideencoded peptidomes. Nat Protoc. 2019;14(8):2596.

29. Xu GJ, Kula T, Xu Q, Li MZ, Vernon SD, Ndung'u T, et al. Viral immunology. Comprehensive serological profiling of human populations using a synthetic human virome. Science. 2015;348(6239):aaa0698.

30. Khan T, Rahman M, Ali FA, Huang SSY, Ata M, Zhang Q, et al. Distinct antibody repertoires against endemic human coronaviruses in children and adults. JCI Insight. 2021;6(4).

31. Mina MJ, Kula T, Leng Y, Li M, de Vries RD, Knip M, et al. Measles virus infection diminishes preexisting antibodies that offer protection from other pathogens. Science. 2019;366(6465):599-606.

32. Hasan MR, Rahman M, Khan T, Saeed A, Sundararaju S, Flores A, et al. Virome-wide serological profiling reveals association of herpesviruses with obesity. Sci Rep. 2021;11(1):2562. 
663

664

665

666

667

668

669

670

671

672

673

674

675

676

677

678

679

680

681

682

683

684

33. Halacli SO, Ayvaz DC, Sun-Tan C, Erman B, Uz E, Yilmaz DY, et al. STK4 (MST1) deficiency in two siblings with autoimmune cytopenias: A novel mutation. Clin Immunol. 2015;161(2):316-23.

34. Hong JJ, Amancha PK, Rogers K, Ansari AA, Villinger F. Re-evaluation of PD-1 expression by T cells as a marker for immune exhaustion during SIV infection. PLoS One. 2013;8(3):e60186.

35. Hofmeyer KA, Jeon $\mathrm{H}$, Zang X. The PD-1/PD-L1 (B7-H1) pathway in chronic infectioninduced cytotoxic T lymphocyte exhaustion. J Biomed Biotechnol. 2011;2011:451694.

36. Dong Y, Li X, Zhang L, Zhu Q, Chen C, Bao J, et al. CD4(+) T cell exhaustion revealed by high PD-1 and LAG-3 expression and the loss of helper T cell function in chronic hepatitis B. BMC Immunol. 2019;20(1):27.

37. Grywalska E, Smarz-Widelska I, Korona-Glowniak I, Mertowski S, Gosik K, Hymos A, et al. PD-1 and PD-L1 Expression on Circulating Lymphocytes as a Marker of Epstein-Barr Virus Reactivation-Associated Proliferative Glomerulonephritis. Int J Mol Sci. $2020 ; 21(21)$.

38. Cichocki F, Grzywacz B, Miller JS. Human NK Cell Development: One Road or Many? Front Immunol. 2019;10:2078.

39. Poli A, Michel T, Theresine M, Andres E, Hentges F, Zimmer J. CD56bright natural killer (NK) cells: an important NK cell subset. Immunology. 2009;126(4):458-65.

40. Gujer C, Murer A, Muller A, Vanoaica D, Sutter K, Jacque E, et al. Plasmacytoid dendritic cells respond to Epstein-Barr virus infection with a distinct type I interferon subtype profile. Blood Adv. 2019;3(7):1129-44. 
41. Moran I, Avery DT, Payne K, Lenthall H, Davies EG, Burns S, et al. B cell-intrinsic requirement for STK4 in humoral immunity in mice and human subjects. J Allergy Clin Immunol. 2019;143(6):2302-5.

42. Pollard AJ, Bijker EM. Publisher Correction: A guide to vaccinology: from basic principles to new developments. Nat Rev Immunol. 2021;21(2):129.

43. Boisson-Dupuis S, Ramirez-Alejo N, Li Z, Patin E, Rao G, Kerner G, et al. Tuberculosis and impaired IL-23-dependent IFN-gamma immunity in humans homozygous for a common TYK2 missense variant. Sci Immunol. 2018;3(30).

44. Frederico B, May JS, Efstathiou S, Stevenson PG. BAFF receptor deficiency limits gammaherpesvirus infection. J Virol. 2014;88(8):3965-75.

45. Kuo SH, Yeh PY, Chen LT, Wu MS, Lin CW, Yeh KH, et al. Overexpression of B cellactivating factor of TNF family (BAFF) is associated with Helicobacter pyloriindependent growth of gastric diffuse large B-cell lymphoma with histologic evidence of MALT Iymphoma. Blood. 2008;112(7):2927-34.

46. Novak AJ, Grote DM, Stenson M, Ziesmer SC, Witzig TE, Habermann TM, et al. Expression of BLyS and its receptors in B-cell non-Hodgkin lymphoma: correlation with disease activity and patient outcome. Blood. 2004;104(8):2247-53.

47. Oki Y, Georgakis GV, Migone TS, Kwak LW, Younes A. Prognostic significance of serum B-lymphocyte stimulator in Hodgkin's lymphoma. Haematologica. 2007;92(2):269-70.

48. Onda K, lijima K, Katagiri YU, Okita H, Saito M, Shimizu T, et al. Differential effects of BAFF on B cell precursor acute lymphoblastic leukemia and Burkitt lymphoma. Int J Hematol. 2010;91(5):808-19. 
49. Ma SD, Xu X, Jones R, Delecluse HJ, Zumwalde NA, Sharma A, et al. PD-1/CTLA-4 Blockade Inhibits Epstein-Barr Virus-Induced Lymphoma Growth in a Cord Blood Humanized-Mouse Model. PLoS Pathog. 2016;12(5):e1005642.

711 50. Inthasot V, Bruyneel M, Muylle I, Ninane V. Severe pulmonary infections complicating nivolumab treatment for lung cancer: a report of two cases. Acta Clin Belg. 2020;75(4):308-10.

714 51. Barber DL, Sakai S, Kudchadkar RR, Fling SP, Day TA, Vergara JA, et al. Tuberculosis following PD-1 blockade for cancer immunotherapy. Sci Transl Med. 2019;11(475).

52. Anastasopoulou A, Ziogas DC, Samarkos M, Kirkwood JM, Gogas H. Reactivation of tuberculosis in cancer patients following administration of immune checkpoint inhibitors: current evidence and clinical practice recommendations. J Immunother Cancer. 2019;7(1):239. 
A

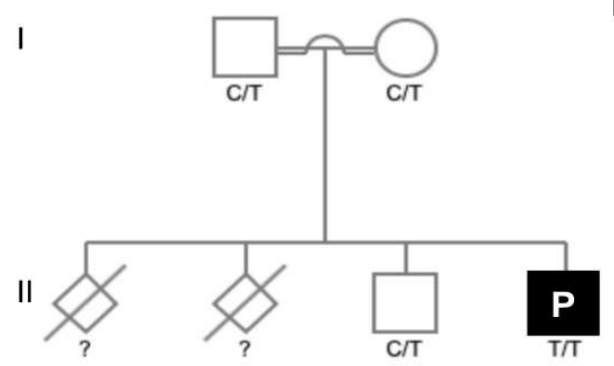

B

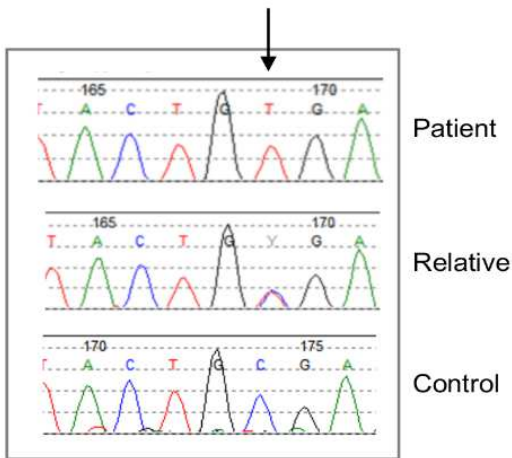

C

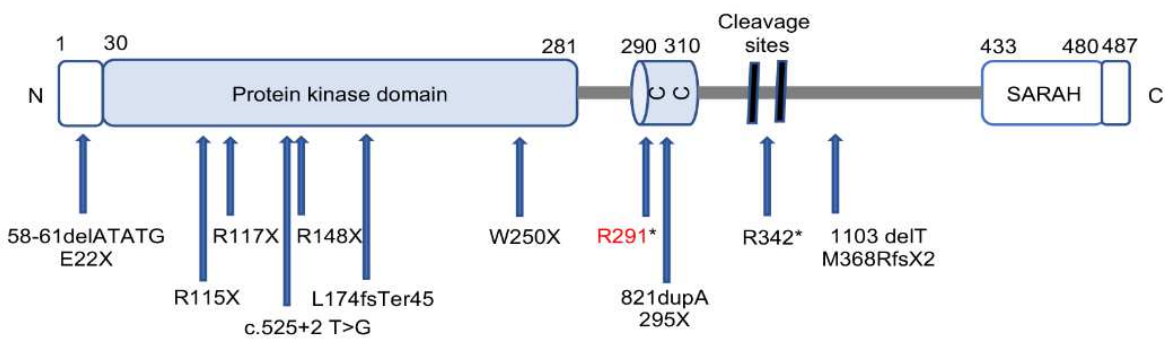

D

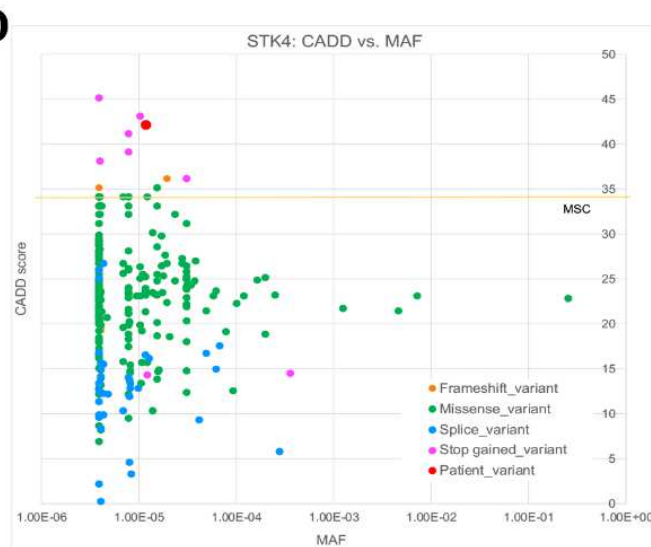

E

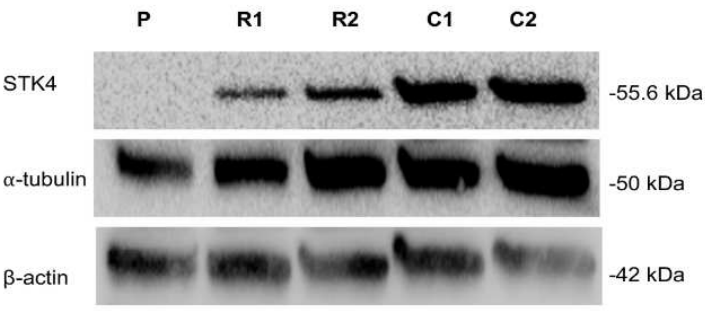

Figure 1 Identification of a homozygous STK4 gene mutation in a patient from consanguineous parents. (A) Pedigree and segregation of the STK4 gene mutation. The patient $(P)$ is homozygous for the mutation. Question marks (?) indicate individuals whose genetic status could not be evaluated. (B) Electropherograms of partial sequences of STK4 corresponding to the mutation in a healthy control (bottom), patient (up) and one of the patient's STK $4^{\text {thtmut }}$ relatives (middle), representative for any of the three healthy family members. The reference vs. altered nucleotide position is indicated by a black arrow. (C) Schematic illustration of the protein encoded by the STK4 gene, with domain boundaries and other features retrieved from the UniProtKB (www.uniprot.org) (entry Q13043). Blue arrows indicate previously reported variants $(2-5,9-12,15,33)$. The variant of the patient in the present study is indicated in red text. CC, coiled coil domain; SARAH, Sav/Rassf/Hpo domain (IPR024205). (D) Combined annotation-dependent depletion score (CADD) serves as a measure to predict the functional impact of the variant. Data from the gnomAD database were used to plot minor allele frequency (MAF) against the CADD score values of all known variants in STK4 and the patient's variant. (E) Western blot analysis of STK4 protein expression in PBMC-derived T lymphocytes from the patient $(P)$, two STK4 ${ }^{\text {wt/mut }}$ heterozygeous relatives (R1 and R2) and two unrelated STK4 ${ }^{\text {wtwt }}$, healthy controls (C1 and C2); $\alpha$-tubulin and $\beta$-actin antibodies were used as controls. 

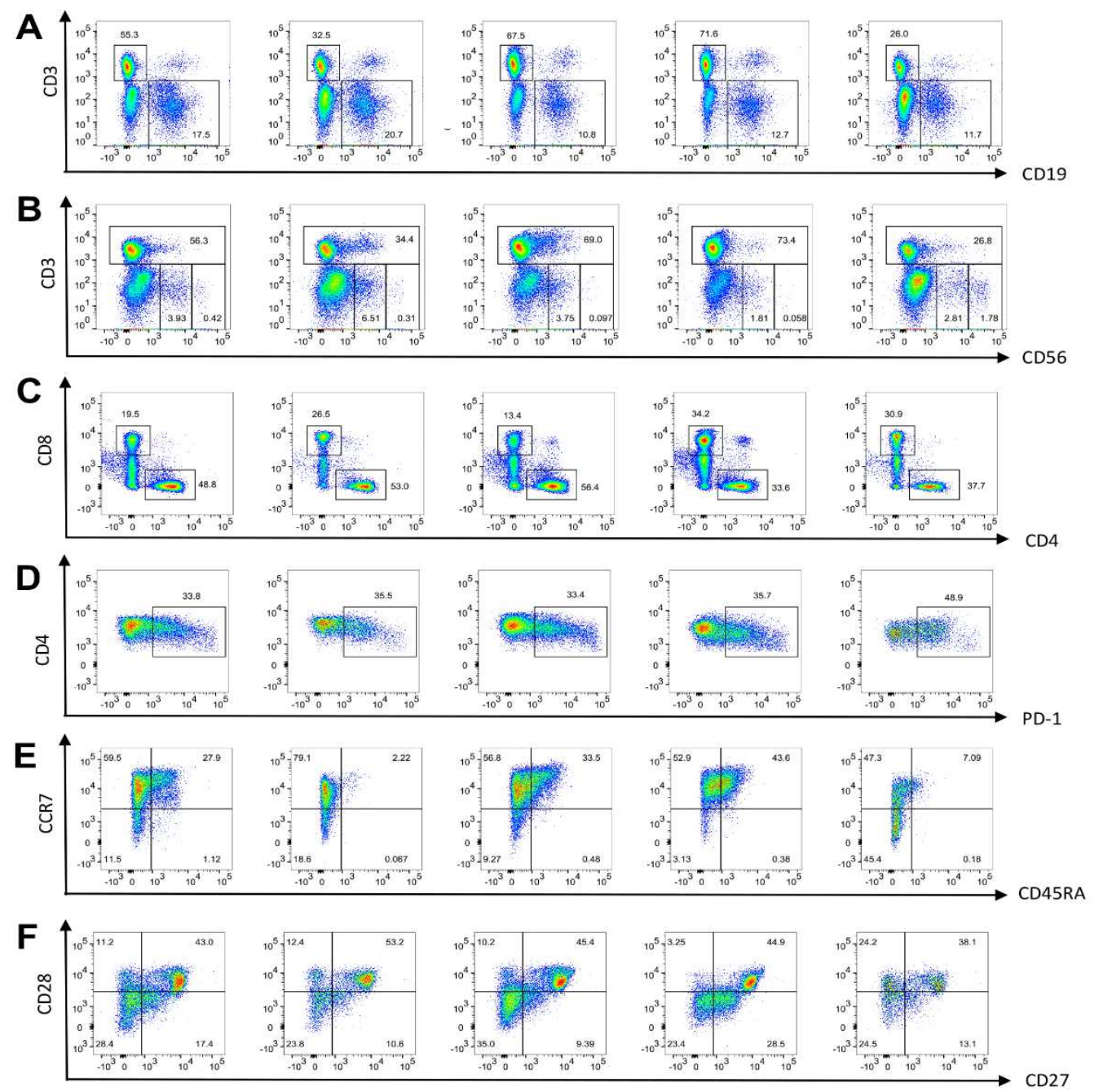

741
G

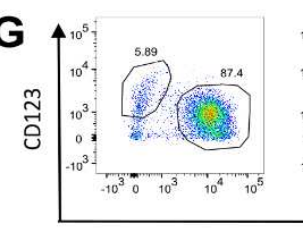

Control

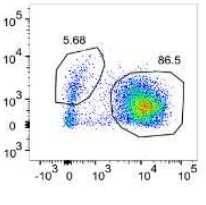

Relative 1

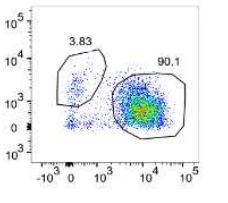

Relative 2

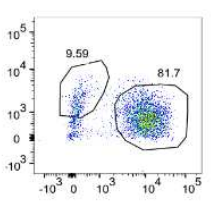

Relative 3

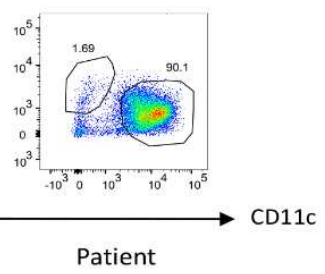

Figure 2 Leukocyte subsets in the STK4-deficient patient, his parents and sibling, and one unrelated healthy control. For all experiments, subjects are presented in the following order from left to right: Unrealted control, the patient's three relatives, and the patient. (A) Frequency of $\mathrm{B}\left(\mathrm{CD}^{-} \mathrm{CD} 19^{+}\right)$and $\mathrm{T}$ lymphocytes $\left(\mathrm{CD}^{+} \mathrm{CD} 19^{-}\right)$among $\mathrm{CD}^{+} 5^{+}$ lymphocytes. (B) Frequency of $\mathrm{T}$ lymphocytes $\left(\mathrm{CD}^{+}\right)$and $\mathrm{NK}$ cell immunophenotyping, showing the frequency of CD56 $6^{\text {bright }}\left(C D 3^{+} C D 56^{\text {bright }}\right)$ and CD56 $6^{\text {dim }}\left(C D 3^{+} C D 56^{\text {dim }}\right)$ NK cells among $\mathrm{CD} 45^{+}$lymphocytes. (C) Frequency of cytotoxic $\left(\mathrm{CD} 3^{+} \mathrm{CD} 8^{+}\right)$and helper $\left(\mathrm{CD} 3^{+} \mathrm{CD} 4^{+}\right)$ $\mathrm{T}$ lymphocytes among the $\mathrm{CD} 3^{+}$lymphocyte subset. (D) Frequency of PD-1 ${ }^{+} \mathrm{T}$ lymphocytes $\left(\mathrm{CD} 4^{+} \mathrm{PD}-1^{+}\right)$among the $\mathrm{CD} 4^{+} \mathrm{T}$ cell subset. (E) Frequency of naïve $\left(\mathrm{CD} 45 \mathrm{RA} \mathrm{A}^{+} \mathrm{CCR} 7^{+}\right)$, central memory (CD45RA $\left.{ }^{-} C C R 7^{+}\right)$, effector memory (CD45RA-CCR7 ${ }^{-}$) and effector memory cells reexpressing CD45RA ( $\left.T_{\text {EMRA }}\right)\left(C D 45 R A^{+} C C R 7^{-}\right)$cells among the $C D 4^{+} T$ cell compartment. $(\mathbf{F})$ Frequency of $\mathrm{CD}_{2} 7^{+}$and $\mathrm{CD}^{2} 8^{+} \mathrm{T}$ helper subsets within the $\mathrm{CD} 4^{+}$compartment. (G) Frequency of myeloid dendritic cells (mDCs) $\left(\mathrm{CD} 123^{-} \mathrm{CD} 11 \mathrm{c}^{+}\right)$and plasmacytoid dendritic cells (pDCs) (CD123 ${ }^{+}$CD11 $\left.c^{-}\right)$among the CD45 ${ }^{+} \mathrm{HLA}^{-} \mathrm{DR}^{+} \mathrm{CD} 3^{-} \mathrm{CD} 14^{-} \mathrm{CD} 19^{-}, \mathrm{CD}^{-} 0^{-} \mathrm{CD}^{-} 6^{-}$dendritic cell population. 

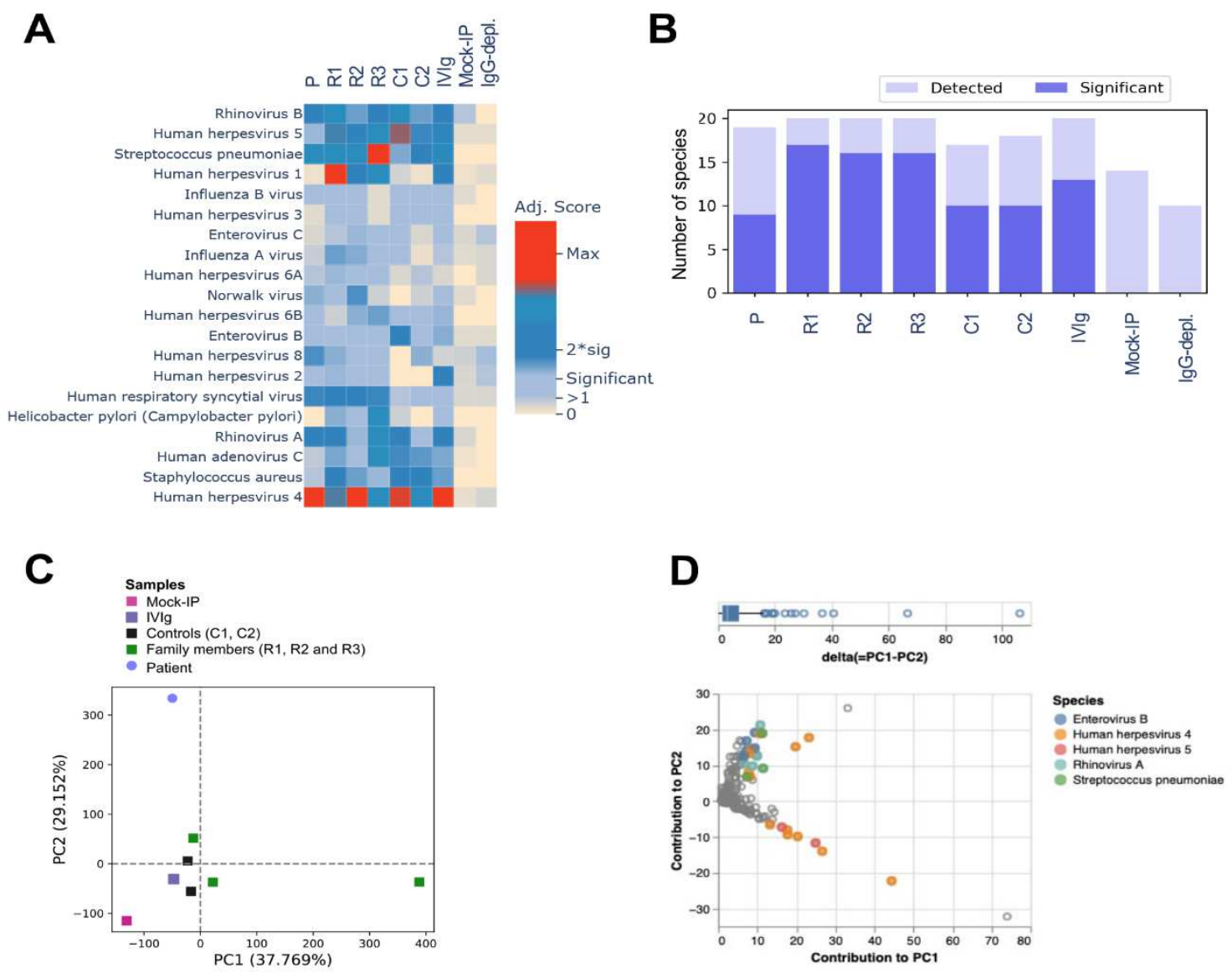

Figure 3 Microbial exposure profile and antiviral antibody repertoire in the STK4deficient patient. (A) Antibody profile in the STK $4^{-/}$patient $(\mathrm{P})$, his STK $4^{\mathrm{WT} /-}$ family members (R1, R2 and R3) as well as two unrelated STK4 ${ }^{\text {WT/WT }}$ controls (C1 and C2). Pooled human plasma used for intravenous immunoglobulin therapy (IVIg), human IgG-depleted serum (IgGdepl.) and mock-IP samples served as additional controls. The heatmap plot shows speciesspecific adjusted score values, which served as a quantitative measure of the number of antibody specificities targeting a given microbial species. (B) Bar plot depicting, for each sample shown in $(\mathbf{A})$, the number of species for which peptides were significantly enriched by PhIP-Seq (i.e., at least one antibody specificity was detected) (light blue) and number of species for which the adjusted virus score values passed the significance cut-off (i.e., the sample was considered seropositive for that given species) (dark blue). (C) Principal component analysis (PCA) of the - $\log _{10}(P$-values) of significantly enriched peptides for each sample as shown in $(\mathbf{A})$. The scatter plot shows the contribution of the significantly enriched peptides to principal component (PC)1 and PC2. (D) Scatter plot showing the contribution of enriched peptides in the patient's sample to PC1 and PC2. Peptides are color-coded by species. Peptides shown in grey correspond to species for which less than two peptides had a delta (PC1-PC2) of more than the 70th percentile (top). 


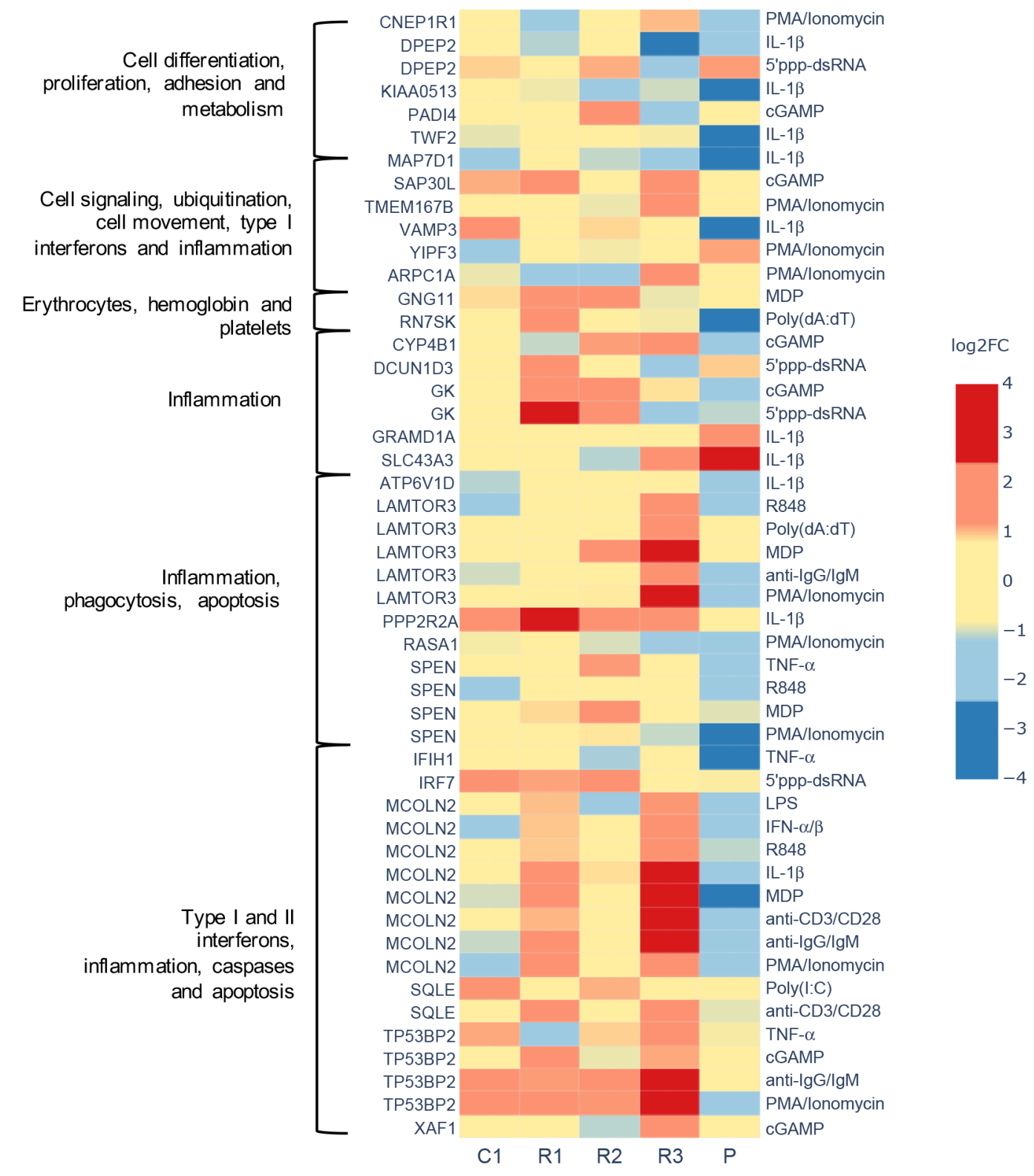

Figure 4 Unique gene expression signature in whole blood samples from the STK4-deficient patient following in vitro stimulation. The heatmap shows the $\log _{2}-$ transformed fold change values (log2FC) of the differentially expressed genes (DEGs) among the 180 target genes for which transcriptional responses in the patient's $(P)$ whole blood samples to in vitro stimulation showed a variance of $|\log 2 \mathrm{FC}|>1$ compared to those of the other family members (R1, R2 and R3) and an unrelated control (C1). Gene-stimuli pairs are grouped according to the functional annotation of the gene cluster as described previously

783 (20). 
A

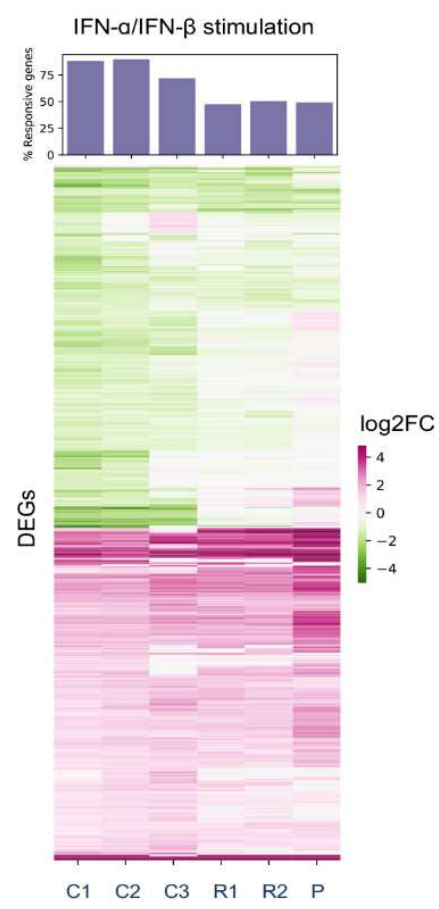

B

IFN- $\alpha /$ IFN- $\beta$ stimulation

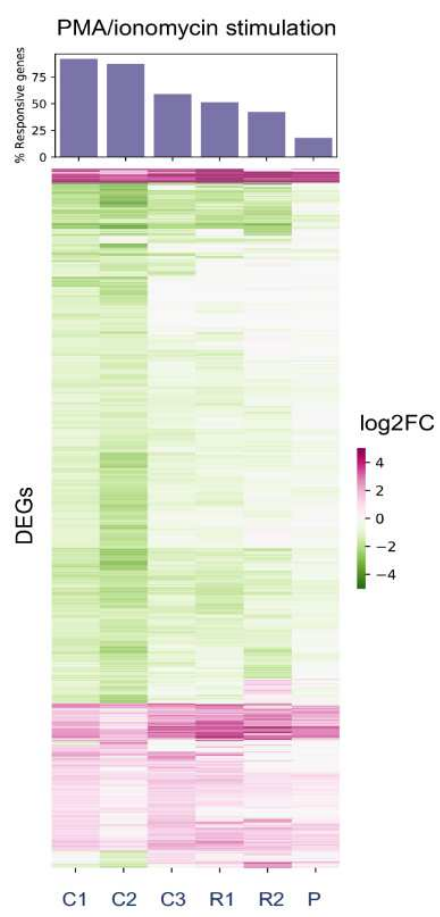

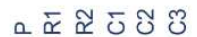

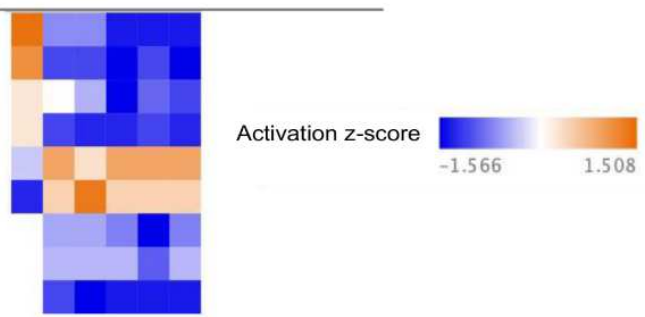

a ᄄᄄ ธิธ

PMA/ionomycin stimulation

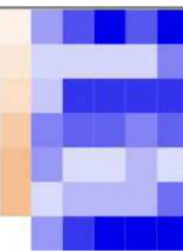

Role of NFAT in Regulation of the Immune Response

B Cell Activating Factor Signaling

NAD Salvage Pathway II

Cytotoxic T Lymphocyte-mediated Apoptosis of Target Cells

p53 Signaling

OX40 Signaling Pathway

Figure $5 \quad$ RNA-Seq and gene enrichment analysis of stimulated PBMCs. (A) The heatmaps show $\log _{2}$-transformed fold change values (log2FC) of differentially expressed genes (DEGs) following in vitro stimulation of PBMCs obtained from the STK4-deficient patient $(P)$, two of his family members (R1 and R2) and three unrelated controls (C1, C2 and C3) with either recombinant human IFN- $\alpha$ and IFN- $\beta$ (left), or PMA and ionomycin (right). Pink indicates activated genes and green indicates repressed genes. (B) Heatmaps show the activation zscore values of a canonical pathway comparison analysis using the QIAGEN Ingenuity Pathway Analysis software for stimulation with recombinant human IFN- $\alpha$ and IFN- $\beta$ (top), or PMA and ionomycin (bottom). Red indicates activated pathways and blue indicates repressed pathways. 


\section{Figures}

A

।

II

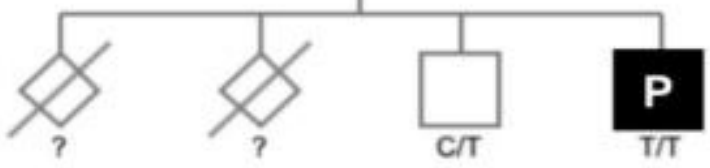

B

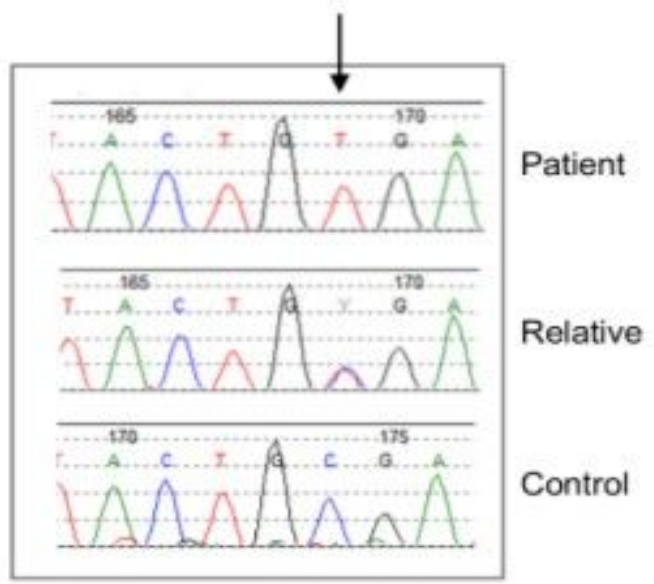

C

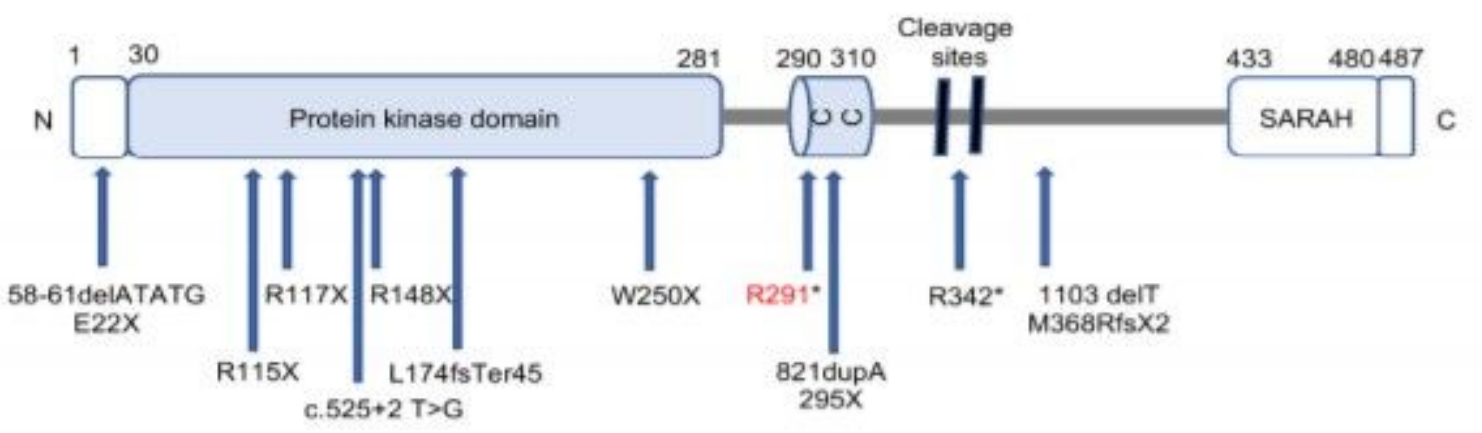

D

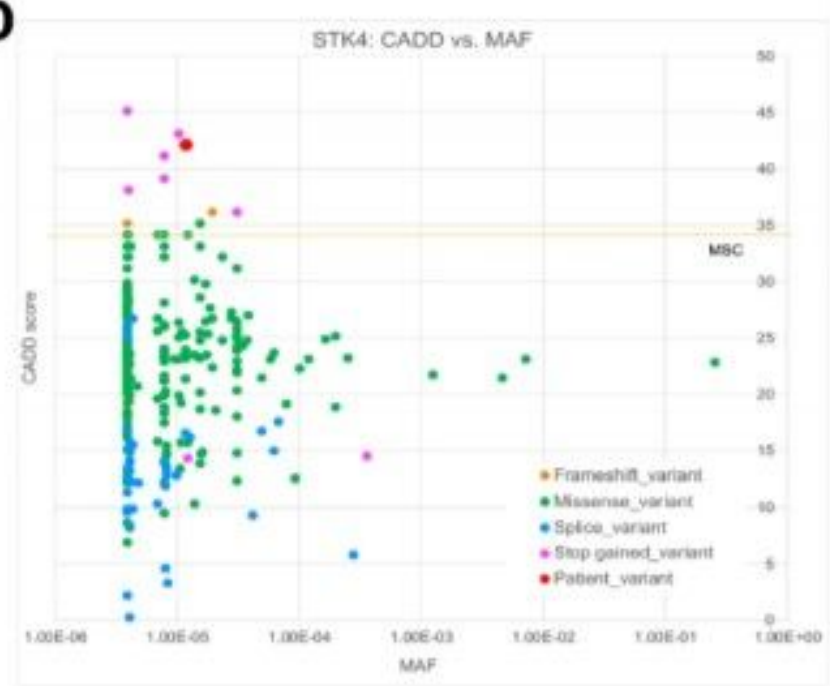

E

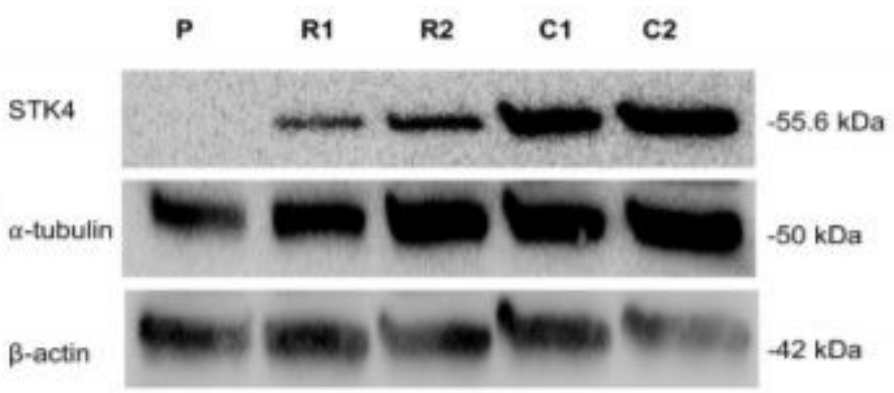

\section{Figure 1}

Identification of a homozygous STK4 gene mutation in a patient from consanguineous parents. (A) Pedigree and segregation of the STK4 gene mutation. The patient $(P)$ is homozygous for the mutation. Question marks (?) indicate individuals whose genetic status could not be evaluated. (B) Electropherograms of partial sequences of STK4 corresponding to the mutation in a healthy control (bottom), patient (up) and one of the patient's STK4wt/mut relatives (middle), representative for any of 
the three healthy family members. The reference vs. altered nucleotide position is indicated by a black arrow. (C) Schematic illustration of the protein encoded by the STK4 gene, with domain boundaries and other features retrieved from the UniProtKB (www.uniprot.org) (entry Q13043). Blue arrows indicate previously reported variants $(2-5,9-12,15,33)$. The variant of the patient in the present study is indicated in red text. CC, coiled coil domain; SARAH, Sav/Rassf/Hpo domain (IPR024205). (D) Combined annotation-dependent depletion score (CADD) serves as a measure to predict the functional impact of the variant. Data from the gnomAD database were used to plot minor allele frequency (MAF) against the CADD score values of all known variants in STK4 and the patient's variant. (E) Western blot analysis of STK4 protein expression in PBMC-derived T lymphocytes from the patient (P), two STK4wt/mut heterozygeous relatives (R1 and R2) and two unrelated STK4wt/wt, healthy controls (C1 and C2); atubulin and b-actin antibodies were used as controls. 
A
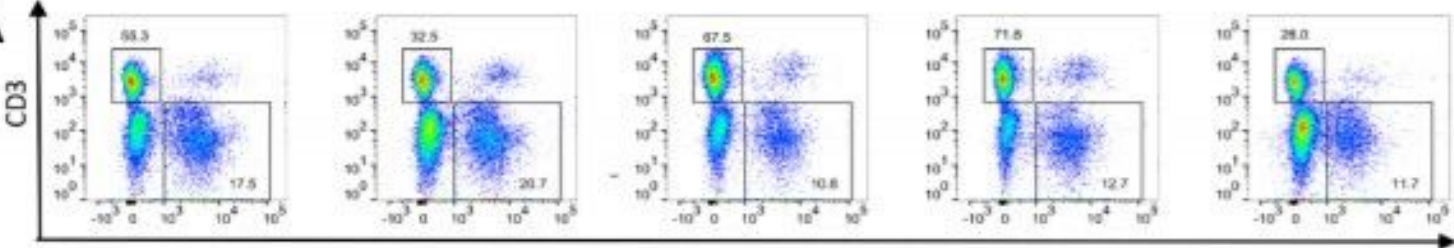

CD19

B
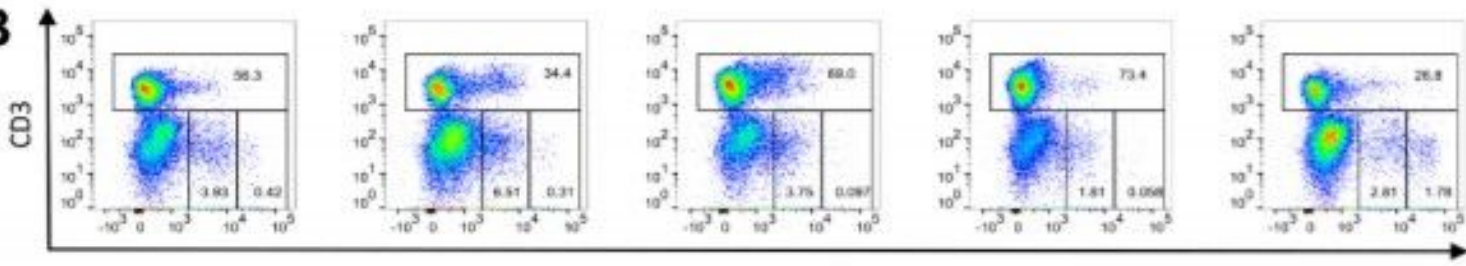

CD56

C
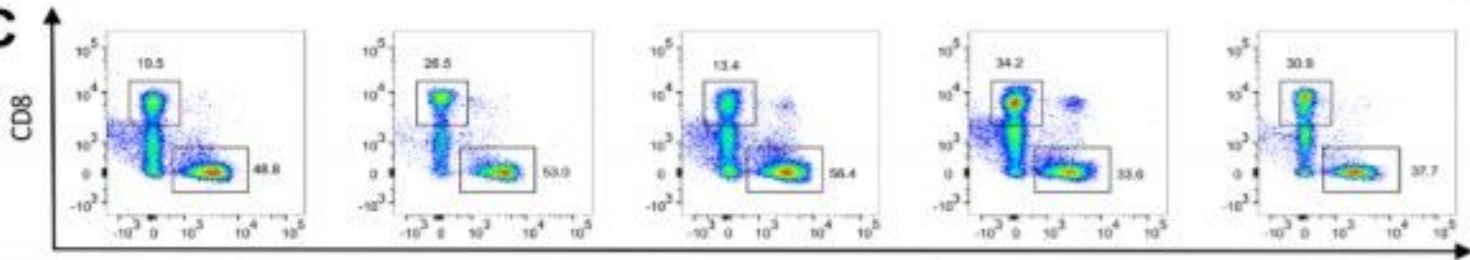

CD4
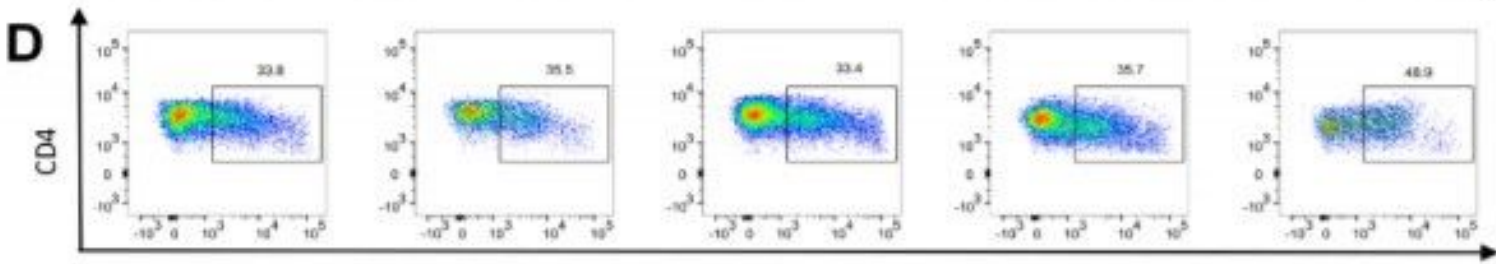

PD-1
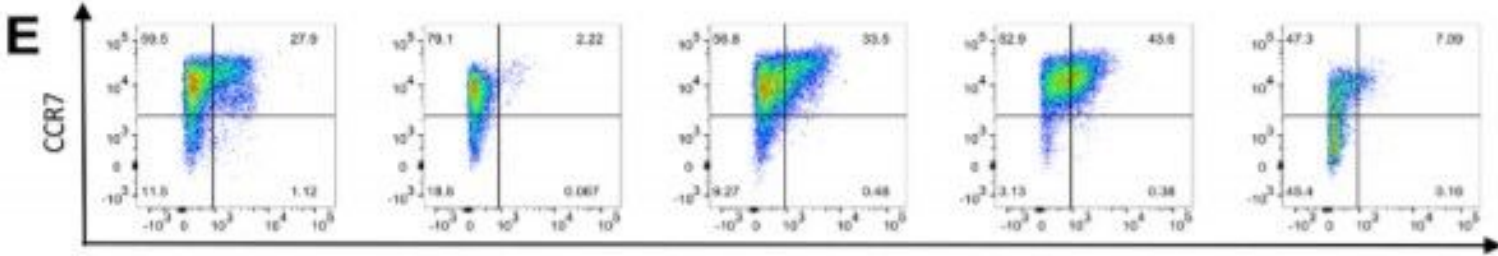

CD45RA
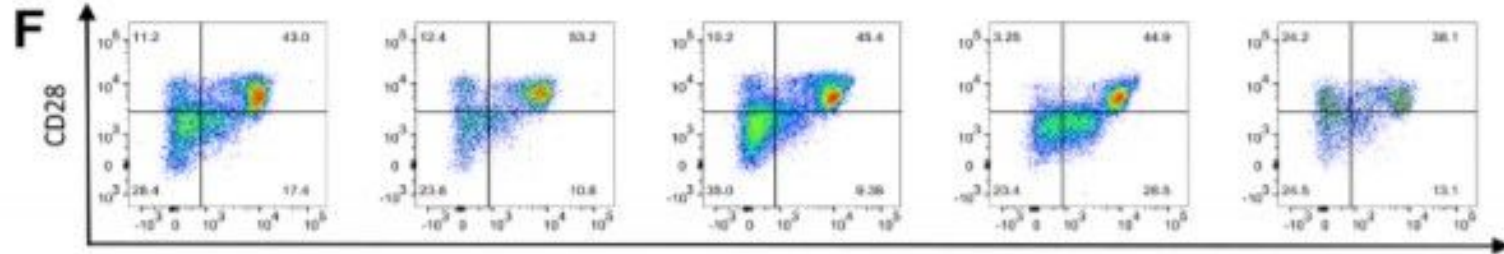

$\mathrm{CD} 27$
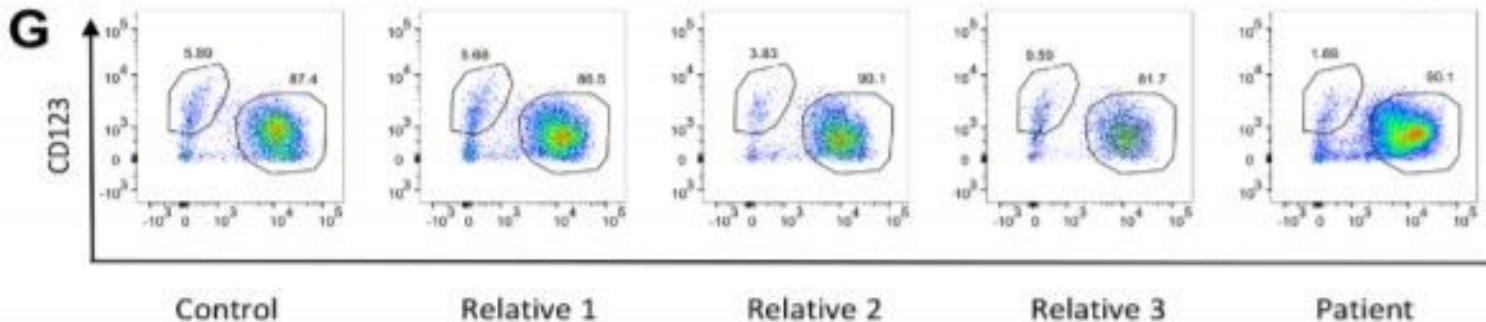

Relative 1

Relative 2

Relative 3

Patient

\section{Figure 2}

Leukocyte subsets in the STK4-deficient patient, his parents and sibling, and one unrelated healthy control. For all experiments, subjects are presented in the following order from left to right: Unrealted control, the patient's three relatives, and the patient. (A) Frequency of B (CD3- CD19+) and T lymphocytes (CD3+ CD19-) among CD45+ lymphocytes. (B) Frequency of T lymphocytes (CD3+) and NK cell immunophenotyping, showing the frequency of CD56bright (CD3+ CD56bright) and CD56dim (CD3+ 
CD56dim ) NK cells among CD45+ lymphocytes. (C) Frequency of cytotoxic (CD3+ CD8+) and helper (CD3+ CD4+ ) T lymphocytes among the CD3+ lymphocyte subset. (D) Frequency of PD-1+ T lymphocytes (CD4+ PD-1+) among the CD4+ T cell subset. (E) Frequency of naïve (CD45RA+ CCR7+ ), central memory (CD45RACCR7+ ), effector memory (CD45RACCR7- ) and effector memory cells reexpressing CD45RA (TEMRA) (CD45RA+ CCR7-) cells among the CD4+ T cell compartment. (F) Frequency of $\mathrm{CD} 27+$ and $\mathrm{CD} 28+\mathrm{T}$ helper subsets within the CD4+ compartment. (G) Frequency of myeloid dendritic cells (mDCs) (CD123- CD11c+) and plasmacytoid dendritic cells ( $\mathrm{pDCs}$ ) (CD123+ CD11 c- ) among the CD45+ HLA-DR+ CD3- CD14- CD19- , CD20- CD56- dendritic cell population.
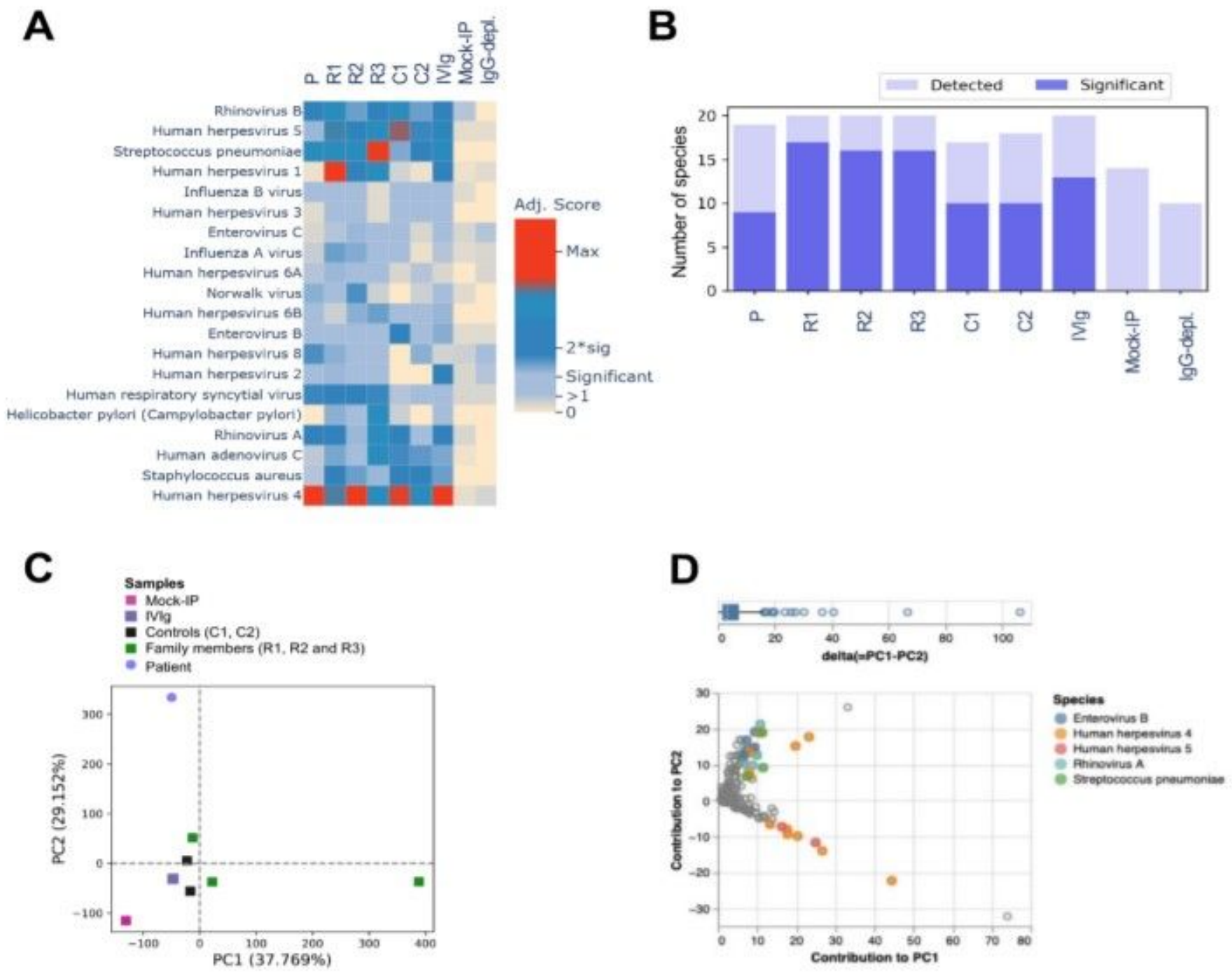

\section{Figure 3}

Microbial exposure profile and antiviral antibody repertoire in the STK4- deficient patient. (A) Antibody profile in the STK4-/- patient (P), his STK4WT/- family members (R1, R2 and R3) as well as two unrelated STK4WT/WT controls (C1 and C2). Pooled human plasma used for intravenous immunoglobulin therapy (IVIg), human IgG-depleted serum (IgG depl.) and mock-IP samples served as additional controls. The heatmap plot shows species specific adjusted score values, which served as a quantitative measure of the number of antibody specificities targeting a given microbial species. (B) Bar plot depicting, for each 
sample shown in (A), the number of species for which peptides were significantly enriched by PhIP-Seq (i.e., at least one antibody specificity was detected) (light blue) and number of species for which the adjusted virus score values passed the significance cut-off (i.e., the sample was considered seropositive for that given species) (dark blue). (C) Principal component analysis (PCA) of the -log10(P-values) of significantly enriched peptides for each sample as shown in (A). The scatter plot shows the contribution of the significantly enriched peptides to principal component (PC)1 and PC2. (D) Scatter plot showing the contribution of enriched peptides in the patient's sample to PC1 and PC2. Peptides are color-coded by species. Peptides shown in grey correspond to species for which less than two peptides had a delta (PC1PC2) of more than the 70th percentile (top). 


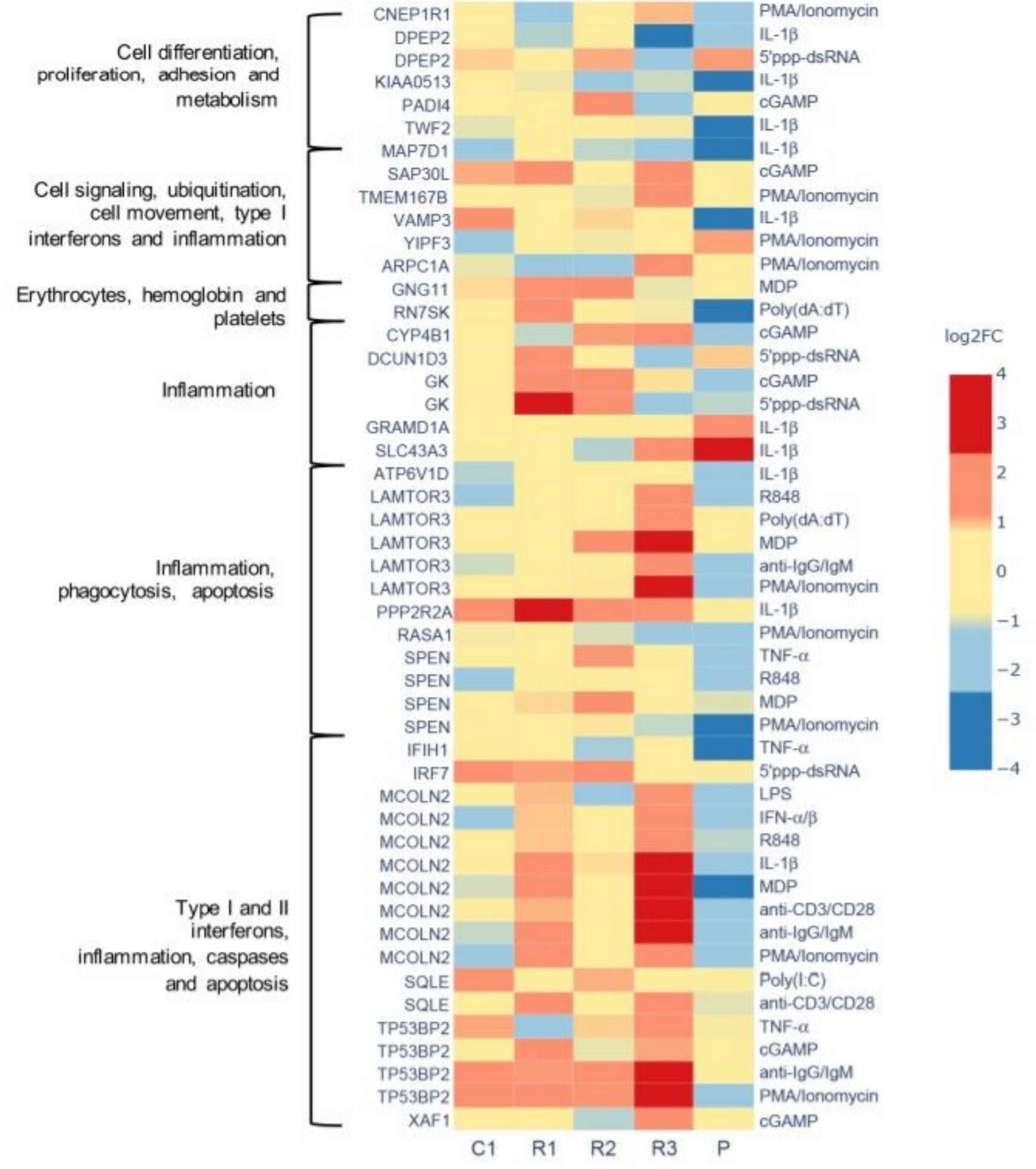

\section{Figure 4}

Unique gene expression signature in whole blood samples from the STK4-deficient patient following in vitro stimulation. The heatmap shows the log2- transformed fold change values (log2 $\mathrm{FC}$ ) of the differentially expressed genes (DEGs) among the 180 target genes for which transcriptional responses in the patient's $(P)$ whole blood samples to in vitro stimulation showed a variance of $\| \log 2 \mathrm{FC} \mid>1$ compared 
to those of the other family members (R1, R2 and R3) and an unrelated control (C1). Gene-stimuli pairs are grouped according to the functional annotation of the gene cluster as described previously (20).

A

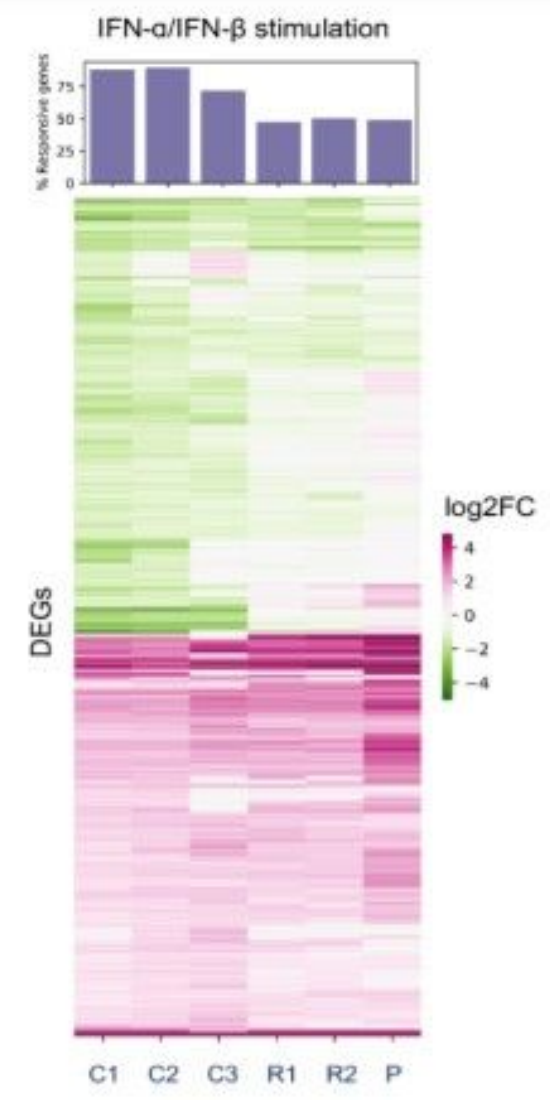

B

IFN-a/IFN- $\beta$ stimulation
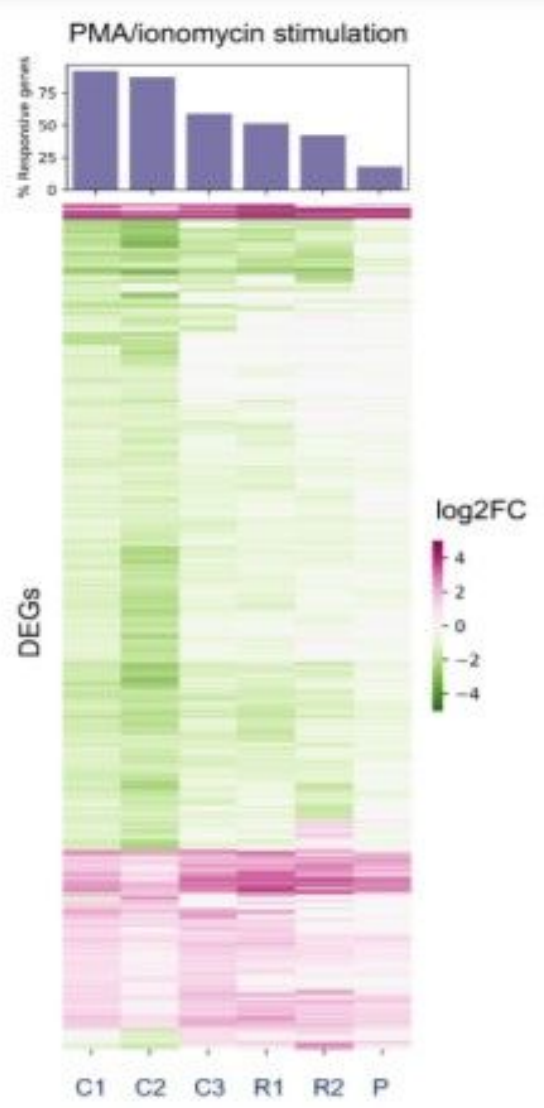

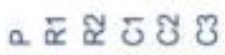

\footnotetext{
Nur 77 Signaling in T Lymphocytes

Calcium-induced T Lymphocyte Apoptosis

Superpathway of Inositol Phosphate Compounds

Systemic Lupus Erythematosus in T Cell Signaling Pathway

SAPK/JNK Signaling

Antiproliferative Role of TOB in T Cell Signaling

Coronavirus Pathogenesis Pathway

IL-23 Signaling Pathway

NRF2-mediated Oxidative Stress Res ponse
}

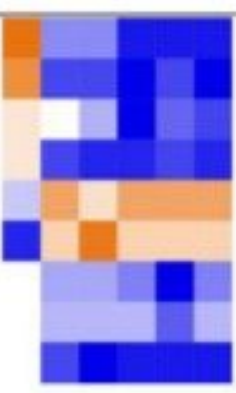

Activation z-score

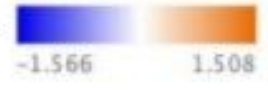

PMA/ionomycin stimulation

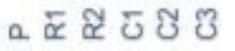

Role of NFAT in Regulation of the Immune Response

B Cell Activating Factor Signaling

NAD Salvage Pathway II

Cytotoxic T Lymphocyte-mediated Apoptosis of Target Cells

p53 Signaling

OX40 Signaling Pathway

Spliceosomal Cycle

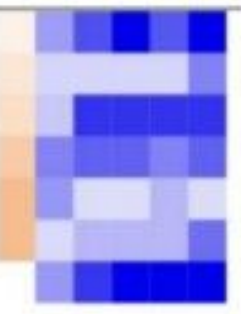

Activation z-score

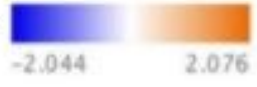

Figure 5

RNA-Seq and gene enrichment analysis of stimulated PBMCs. (A) The heatmaps show log2-transformed fold change values (log2FC) of differentially expressed genes (DEGs) following in vitro stimulation of PBMCs obtained from the STK4-deficient patient (P), two of his family members (R1 and R2) and three 
unrelated controls (C1, C2 and C3) with either recombinant human IFN-a and IFN-b (left), or PMA and ionomycin (right). Pink indicates activated genes and green indicates repressed genes. (B) Heatmaps show the activation z score values of a canonical pathway comparison analysis using the QIAGEN Ingenuity Pathway Analysis software for stimulation with recombinant human IFN-a and IFN-b (top), or PMA and ionomycin (bottom). Red indicates activated pathways and blue indicates repressed pathways.

\section{Supplementary Files}

This is a list of supplementary files associated with this preprint. Click to download.

- STK4SupplementalTablesS3andS42021Mar14.xlsx

- SupplementalMaterialsSTK42021March14final.pdf 